ANALYSIS \& PDE Volume $8 \quad$ No. $7 \quad 2015$ YAIZA CANZANI AND BORIS HANIN SCALING LIMIT FOR THE KERNEL OF THE SPECTRAL PROJECTOR

AND REMAINDER ESTIMATES IN THE POINTWISE WEYL LAW -

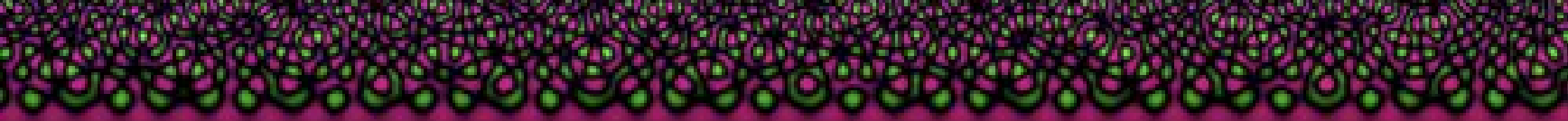




\title{
SCALING LIMIT FOR THE KERNEL OF THE SPECTRAL PROJECTOR AND REMAINDER ESTIMATES IN THE POINTWISE WEYL LAW
}

\author{
YAIZA CANZANI AND BORIS HANIN
}

\begin{abstract}
Let $(M, g)$ be a compact, smooth, Riemannian manifold. We obtain new off-diagonal estimates as $\lambda \rightarrow \infty$ for the remainder in the pointwise Weyl law for the kernel of the spectral projector of the Laplacian onto functions with frequency at most $\lambda$. A corollary is that, when rescaled around a non-self-focal point, the kernel of the spectral projector onto the frequency interval $(\lambda, \lambda+1]$ has a universal scaling limit as $\lambda \rightarrow \infty$ (depending only on the dimension of $M$ ). Our results also imply that, if $M$ has no conjugate points, then immersions of $M$ into Euclidean space by an orthonormal basis of eigenfunctions with frequencies in $(\lambda, \lambda+1]$ are embeddings for all $\lambda$ sufficiently large.
\end{abstract}

\section{Introduction}

Suppose that $(M, g)$ is a smooth, compact, Riemannian manifold without boundary of dimension $n \geq 2$. Let $\Delta_{g}$ be the nonnegative Laplacian acting on $L^{2}(M, g, \mathbb{R})$ and let $\left\{\varphi_{j}\right\}_{j}$ be an orthonormal basis of eigenfunctions:

$$
\Delta_{g} \varphi_{j}=\lambda_{j}^{2} \varphi_{j}
$$

with $0=\lambda_{0}^{2}<\lambda_{1}^{2} \leq \lambda_{2}^{2} \leq \cdots$. This article concerns the $\lambda \rightarrow \infty$ asymptotics of the Schwartz kernel

$$
E_{\lambda}(x, y)=\sum_{\lambda_{j} \leq \lambda} \varphi_{j}(x) \varphi_{j}(y)
$$

of the spectral projection

$$
E_{\lambda}: L^{2}(M, g) \rightarrow \bigoplus_{\mu \in(0, \lambda]} \operatorname{ker}\left(\Delta_{g}-\mu^{2}\right)
$$

onto functions with frequency at most $\lambda$. We are primarily concerned with the behavior of $E_{\lambda}(x, y)$ at points $x, y \in M$ for which the Riemannian distance $\operatorname{dist}_{g}(x, y)$ is less than the injectivity radius $\operatorname{inj}(M, g)$, so that the inverse of the exponential map $\exp _{y}^{-1}(x)$ is well defined. We write

$$
E_{\lambda}(x, y)=\frac{\lambda^{n}}{(2 \pi)^{n}} \int_{|\xi|_{g_{y}}<1} e^{i \lambda\left\langle\exp _{y}^{-1}(x), \xi\right\rangle_{g_{y}}} \frac{d \xi}{\sqrt{\left|g_{y}\right|}}+R(x, y, \lambda),
$$

where the remainder $R(x, y, \lambda)$ is a smooth function of $x$ and $y$. The integral in (3) is over the cotangent fiber $T_{y}^{*} M$ and it is coordinate-independent because the integration measure $d \xi / \sqrt{\left|g_{y}\right|}$ is the quotient

Canzani was partially supported by an NSERC Postdoctoral Fellowship and by NSF grant DMS-1128155. Hanin was partially supported by NSF grant DMS-1400822.

MSC2010: primary 35P20; secondary 58J40, 35L05.

Keywords: spectral projector, pointwise Weyl law, off-diagonal estimates, non-self-focal points . 
of the natural symplectic form $d \xi d y$ on $T^{*} M$ by the Riemannian volume form $\sqrt{\left|g_{y}\right|} d y$. The integral is also symmetric in $x$ and $y$, which can be seen by changing variables from $T_{y}^{*} M$ to $T_{x}^{*} M$ using the parallel transport operator (see (28)).

Our main result, Theorem 2, fits into a long history of estimates on $R(x, y, \lambda)$ as $\lambda \rightarrow+\infty$ (see Section 1.2 for some background). To state it, we need a definition from [Safarov 1988; Sogge and Zelditch 2013]:

Definition 1. A point $x \in M$ is said to be non-self-focal if the set of unit covectors

$$
\mathscr{L}_{x}=\left\{\xi \in S_{x}^{*} M \mid \exp _{x}(t \xi)=x \text { for some } t>0\right\}
$$

has zero measure with respect to the surface measure induced by $g$ on $S_{x}^{*} M$.

Theorem 2. Let $(M, g)$ be a compact, smooth, Riemannian manifold of dimension $n \geq 2$ with no boundary. Suppose $x_{0} \in M$ is a non-self-focal point and let $r_{\lambda}$ be a nonnegative function with $\lim _{\lambda \rightarrow \infty} r_{\lambda}=0$. Then

$$
\sup _{x, y \in B\left(x_{0}, r_{\lambda}\right)}|R(x, y, \lambda)|=o\left(\lambda^{n-1}\right)
$$

as $\lambda \rightarrow \infty$. Here, $B\left(x_{0}, r_{\lambda}\right)$ denotes the geodesic ball of radius $r_{\lambda}$ centered at $x_{0}$ and the rate of convergence depends on $x_{0}$ and $r_{\lambda}$.

The little-o estimate (5) is not new for $x=y$ (i.e., $r_{\lambda}=0$ ). Both Safarov [1988] and Sogge and Zelditch [2002] show that $R(x, x, \lambda)=o\left(\lambda^{n-1}\right)$ when $x$ belongs to a compact subset of the diagonal in $M \times M$ consisting only of non-self-focal points (see also [Safarov and Vassiliev 1997]). Safarov [1988] also obtained $o\left(\lambda^{n-1}\right)$ estimates on $R(x, y, \lambda)$ for $(x, y)$ in a compact subset of $M \times M$ that does not intersect the diagonal (under the assumptions of Theorem 6). Theorem 2 simultaneously allows $x \neq y$ and $\operatorname{dist}_{g}(x, y) \rightarrow 0$ as $\lambda \rightarrow \infty$, closing the gap between the two already-known regimes. We refer the reader to Section 1.2 for further discussion and motivation for Theorem 2 and to Section 2 for an outline of the proof.

An elementary corollary of Theorem 2 is Theorem 3, which gives scaling asymptotics for the Schwartz kernel

$$
E_{(\lambda, \lambda+1]}(x, y):=\sum_{\lambda<\lambda_{j} \leq \lambda+1} \varphi_{j}(x) \varphi_{j}(y)
$$

of the orthogonal projection

$$
E_{(\lambda, \lambda+1]}=E_{\lambda+1}-E_{\lambda}: L^{2}(M, g) \rightarrow \bigoplus_{\mu \in(\lambda, \lambda+1]} \operatorname{ker}\left(\Delta_{g}-\mu^{2}\right) .
$$

Passing to polar coordinates in (3) and using that

$$
\int_{S^{n-1}} e^{i\langle v, \omega\rangle} d \omega=(2 \pi)^{n / 2} \frac{J_{(n-2) / 2}(|v|)}{|v|^{(n-2) / 2}},
$$

it is straightforward to obtain the following result: 
Theorem 3. Let $(M, g)$ be a compact, smooth, Riemannian manifold of dimension $n \geq 2$ with no boundary. Let $x_{0} \in M$ be a non-self-focal point. Consider any nonnegative function $r_{\lambda}$ satisfying $r_{\lambda} \rightarrow 0$ as $\lambda \rightarrow \infty$. Then

$$
\sup _{x, y \in B\left(x_{0}, r_{\lambda}\right)}\left|E_{(\lambda, \lambda+1]}(x, y)-\frac{\lambda^{n-1}}{(2 \pi)^{n / 2}} \frac{J_{(n-2) / 2}\left(\lambda \operatorname{dist}_{g}(x, y)\right)}{\left(\lambda \operatorname{dist}_{g}(x, y)\right)^{(n-2) / 2}}\right|=o\left(\lambda^{n-1}\right),
$$

where $J_{v}$ is the Bessel function of the first kind with index $v, B\left(x_{0}, r_{\lambda}\right)$ denotes the geodesic ball of radius $r_{\lambda}$ centered at $x_{0}$, and dist $_{g}$ is the Riemannian distance.

Remark 4. Under the assumptions of Theorem 3, relation (8) holds for $E_{(\lambda, \lambda+\delta]}$ with any $\delta>0$. The difference is that the Bessel function term is multiplied by $\delta$ and that the rate of convergence depends on $\delta$. Our proof of Theorem 3 is insensitive to the choice of $\delta$.

In normal coordinates at $x_{0},(8)$ therefore implies

$$
\sup _{|u|,|v|<r_{0}}\left|E_{(\lambda, \lambda+1]}\left(x_{0}+\frac{u}{\lambda}, x_{0}+\frac{v}{\lambda}\right)-\frac{\lambda^{n-1}}{(2 \pi)^{n}} \int_{S^{n-1}} e^{i\langle u-v, w\rangle} d \omega\right|=o\left(\lambda^{n-1}\right)
$$

as $\lambda \rightarrow \infty$. The measure $d \omega$ is the Euclidean surface measure on the unit sphere $S^{n-1}$ and the rate of convergence of the error term depends on $r_{0}$ and the point $x_{0}$. The integral over $S^{n-1}$ in (9) is the kernel of the spectral projector onto the generalized eigenspace of eigenvalue 1 for the flat Laplacian on $\mathbb{R}^{n}$ (see [Helgason 1981; Zelditch 2008, §2.1]).

We believe (5) holds for any number of covariant derivatives $\nabla_{x}^{j} \nabla_{y}^{k}$ of the remainder $R(x, y, \lambda)$ with $o\left(\lambda^{n-1}\right)$ replaced by $o\left(\lambda^{n-1+j+k}\right)$. This would immediately imply that the $C^{0}$ convergence in (8) can be upgraded to $C^{k}$ convergence for all $k$. Proving this is work in progress by the authors. Since $E_{(\lambda, \lambda+1]}$ is the covariance kernel for asymptotically fixed frequency random waves on $M$ (see [Sarnak and Wigman 2014; Sodin 2012; Zelditch 2009]), this $C^{\infty}$ convergence would show that the integral statistics of monochromatic random waves near a non-self-focal point depend only on the dimension of $M$. We refer the reader to Section 1.3 for further discussion and motivation for Theorem 3.

1.1. Applications. Combining Theorem 2 with prior results of Safarov [1988], we obtain little-o estimates on $R(x, y, \lambda)$ without requiring $x$ or $y$ to be in a shrinking neighborhood of a single nonfocal point. We recall the following definition from [Safarov 1988; Sogge and Zelditch 2013]:

Definition 5. Let $(M, g)$ be a Riemannian manifold. We say that $x, y \in M$ are mutually nonfocal if the set of unit covectors

$$
\mathscr{L}(x, y)=\left\{\xi \in S_{x}^{*} M \mid \exp _{x}(t \xi)=y \text { for some } t>0\right\}
$$

has zero measure with respect to the Euclidean surface measure induced by $g$ on $S_{x}^{*} M$.

Theorem 6. Let $(M, g)$ be a compact, smooth, Riemannian manifold of dimension $n \geq 2$ with no boundary. Consider any compact set $K \subseteq M \times M$ such that, if $(x, y) \in K$, then $x$ and $y$ are mutually nonfocal and either $x$ or $y$ is a non-self-focal point. Then, as $\lambda \rightarrow \infty$, we have

$$
\sup _{(x, y) \in K}|R(x, y, \lambda)|=o\left(\lambda^{n-1}\right) .
$$


Remark 7. Theorem 6 applies with $K=M \times M$ if $(M, g)$ has no conjugate points.

Theorem 6-proved in Section 7 - can be applied to studying immersions of $(M, g)$ into Euclidean space by arrays of high-frequency eigenfunctions. Let $\left\{\varphi_{j_{1}}, \ldots, \varphi_{j_{m_{\lambda}}}\right\}$ be an orthonormal basis for $\bigoplus_{\lambda<\mu \leq \lambda+1} \operatorname{ker}\left(\Delta_{g}-\mu^{2}\right)$ and consider the maps

$$
\Psi_{(\lambda, \lambda+1]}: M \rightarrow \mathbb{R}^{m_{\lambda}}, \quad \Psi_{(\lambda, \lambda+1]}(x)=\sqrt{\frac{(2 \pi)^{n}}{2 \lambda^{n-1}}}\left(\varphi_{j_{1}}(x), \ldots, \varphi_{j_{m_{\lambda}}}(x)\right) .
$$

The $\lambda^{-(n-1) / 2}$ normalization is chosen so that the diameter of $\Psi_{(\lambda, \lambda+1]}(M)$ in $\mathbb{R}^{m_{\lambda}}$ is bounded above and below as $\lambda \rightarrow \infty$. Maps related to $\Psi_{\lambda}$ are studied in [Bérard et al. 1994; Jones et al. 2008; Potash 2014; Zelditch 2009]. In particular, Zelditch [2009, Proposition 2.3] showed that the maps $\Psi_{(\lambda, \lambda+1]}$ are almost-isometric immersions for large $\lambda$, in the sense that a certain rescaling of the pullback $\Psi_{\lambda}^{*}\left(g_{\text {euc }}\right)$ of the Euclidean metric on $\mathbb{R}^{m_{\lambda}}$ converges pointwise to $g$. A consequence of Theorem 6 is that these maps are actually embeddings for $\lambda$ sufficiently large:

Theorem 8. Let $(M, g)$ be a compact, smooth, Riemannian manifold of dimension $n \geq 2$ with no boundary. If every point $x \in M$ is non-self-focal and all pairs $x, y \in M$ are mutually nonfocal, then there exists $\lambda_{0}>0$ such that the maps $\Psi_{(\lambda, \lambda+1]}: M \rightarrow \mathbb{R}^{m_{\lambda}}$ are embeddings for all $\lambda \geq \lambda_{0}$.

We prove Theorem 8 in Section 7. Note that this result does not hold on the round spheres $S^{n} \subseteq \mathbb{R}^{n+1}$, since even spherical harmonics take on equal values at antipodal points. Since $\Psi_{(\lambda, \lambda+1]}$ are embeddings for $\lambda$ large, it is natural to study $\Psi_{(\lambda, \lambda+1]}(M)$ as a metric space equipped with the distance, dist $\lambda$, induced by the embedding:

$$
\begin{aligned}
\operatorname{dist}_{\lambda}^{2}(x, y): & =\left\|\Psi_{(\lambda, \lambda+1]}(x)-\Psi_{(\lambda, \lambda+1]}(y)\right\|_{l^{2}\left(\mathbb{R}^{m}\right)}^{2} \\
& =\frac{(2 \pi)^{n}}{2 \lambda^{n-1}}\left(E_{(\lambda, \lambda+1]}(x, x)+E_{(\lambda, \lambda+1]}(y, y)-2 E_{(\lambda, \lambda+1]}(x, y)\right) .
\end{aligned}
$$

Theorem 9, also proved in Section 7, gives precise asymptotics for $\operatorname{dist}_{\lambda}(x, y)$ in terms of $\operatorname{dist}_{g}(x, y)$ :

Theorem 9. Let $(M, g)$ be a compact, smooth, Riemannian manifold of dimension $n \geq 2$ with no boundary. Suppose further that every $x \in M$ is non-self-focal and all pairs $x, y \in M$ are mutually nonfocal. As $\lambda \rightarrow \infty$, we have

$$
\sup _{x, y \in M}\left|\frac{1}{\lambda^{2} \operatorname{dist}_{g}^{2}(x, y)}\left[\operatorname{dist}_{\lambda}^{2}(x, y)-\left(\operatorname{vol}\left(S^{n-1}\right)-(2 \pi)^{n / 2} \frac{J_{(n-2) / 2}\left(\lambda \operatorname{dist}_{g}(x, y)\right)}{\left(\lambda \operatorname{dist}_{g}(x, y)\right)^{(n-2) / 2}}\right)\right]\right|=o(1) \text {. }
$$

1.2. Discussion of Theorem 2. Theorem 2 is an extension of Hörmander's pointwise Weyl law [1968, Theorem 4.4]. Hörmander proved that there exists $\varepsilon>0$ such that, if the Riemannian distance $\operatorname{dist}_{g}(x, y)$ between $x$ and $y$ is less than $\varepsilon$, then

$$
E_{\lambda}(x, y)=\frac{\lambda^{n}}{(2 \pi)^{n}} \int_{|\xi|_{g_{y}}<1} e^{i \lambda \psi(x, y, \xi)} \frac{d \xi}{\sqrt{\left|g_{y}\right|}}+O\left(\lambda^{n-1}\right),
$$

where, in Hörmander's terminology, the phase function $\psi$ is adapted to the principal symbol $|\xi|_{g_{y}}$ of $\sqrt{\Delta_{g}}$. After his Theorem 4.4, Hörmander [1968] remarks that the choice of $\psi$ is not unique. However, every 
adapted phase function satisfies

$$
\psi(x, y, \xi)=\langle x-y, \xi\rangle+O\left(|x-y|^{2}|\xi|\right)
$$

In particular, since $\left\langle\exp _{y}^{-1}(x), \xi\right\rangle_{g_{y}}=\langle x-y, \xi\rangle+O\left(|x-y|^{2}|\xi|\right)$, Taylor-expanding (15) yields, for any $r_{0}>0$,

$$
\sup _{\operatorname{dist} g(x, y)<r_{0} / \lambda}\left|E_{\lambda}(x, y)-\frac{\lambda^{n}}{(2 \pi)^{n}} \int_{|\xi|_{g_{y}}<1} e^{i \lambda\left\langle\exp _{y}^{-1}(x), \xi\right\rangle_{g_{y}}} \frac{d \xi}{\sqrt{\left|g_{y}\right|}}\right|=O\left(\lambda^{n-1}\right) .
$$

Changing from one adapted phase to another produces, a priori, an error of $O\left(\lambda^{n-1}\right)$ in (15). With the additional assumption that $x$ and $y$ are near a non-self-focal point, Theorem 2 therefore extends Hörmander's result in two ways. First, our careful choice of phase function $\left\langle\exp _{y}^{-1}(x), \xi\right\rangle_{g_{y}}$ allows us to obtain a $o\left(\lambda^{n-1}\right)$ estimate on $R$ while keeping the amplitude equal to 1 . Second, we allow $\operatorname{dist}_{g}(x, y)$ to shrink arbitrarily slowly with $\lambda$.

Hörmander's phase functions $\psi(x, y, \xi)$ are difficult to analyze directly when $x \neq y$, since they are the solutions to certain Hamilton-Jacobi equations (see [Hörmander 1968, Definition 3.1; 1985b, (29.1.7)]) which we cannot describe explicitly. Instead, in proving Theorem 2, we use a parametrix for the half-wave operator $U(t)=e^{-i t \sqrt{\Delta_{g}}}$ with the geometric phase function $\phi: \mathbb{R} \times M \times T^{*} M: \rightarrow \mathbb{R}$ given by $\phi(t, x, y, \xi)=\left\langle\exp _{y}^{-1}(x), \xi\right\rangle-t|\xi|_{g_{y}}$. Such a parametrix was previously used by Zelditch [2009], where a construction for the amplitude was omitted. Our construction, given in Section 3, makes clear the off-diagonal behavior of $E_{\lambda}(x, y)$ and uses the results of Laptev, Safarov and Vassiliev [Laptev et al. 1994], who treat Fourier integral operators (FIOs) with global phase functions.

Using the phase function $\phi$ simplifies our computations considerably, since the half-density factor $\sqrt{\operatorname{det} \phi_{x, \xi}(t, x, y, \xi)}$, which comes up in the usual parametrix construction for $U(t)$ acting on halfdensities, is independent of $t$ and $\xi$. This makes it easy to obtain the amplitude in a parametrix for $U(t)$ acting on functions from that of $U(t)$ acting on half-densities. For more details, see the outline of the proof of Theorem 2 given in Section 2, as well as Section 3, especially (37).

The error estimate in (15) is sharp on Zoll manifolds (see [Zelditch 1997]), such as the round sphere. The majority of the prior estimates on $R(x, y, \lambda)$ actually treat the case $x=y$. Notably, Bérard [1977] showed that on all compact manifolds of dimension $n \geq 3$ with nonpositive sectional curvatures and on all Riemannian surfaces without conjugate points we have $R(x, x, \lambda)=O\left(\lambda^{n} / \log \lambda\right)$. The $O\left(\lambda^{n-1}\right)$ error in the Weyl asymptotics for the spectral counting function

$$
\#\left\{j: \lambda_{j} \in[0, \lambda]\right\}=\int_{M} E_{\lambda}(x, x) d v_{g}(x)=\left(\frac{\lambda}{2 \pi}\right)^{n} \operatorname{vol}_{g}(M) \cdot \operatorname{vol}_{\mathbb{R}^{n}}\left(B_{1}\right)+\int_{M} R(x, x, \lambda) d v_{g}(x)
$$

has also been improved under various assumptions on the structure of closed geodesics on $(M, g)$ (see [Bérard 1977; Colin de Verdière 1980; Duistermaat and Guillemin 1975; Ivriǔ 1984; Nicolaescu 2012; Petridis and Toth 2002; Randol 1981; Safarov and Vassiliev 1997]). For instance, [Duistermaat and Guillemin 1975; Ivriǔ 1984] prove that $\int_{M} R(x, x, \lambda) d v_{g}(x)=o\left(\lambda^{n-1}\right)$ if $(M, g)$ is aperiodic (i.e., the set of all closed geodesics has measure zero in $\left.S^{*} M\right)$. 
Also related to this article are lower bounds for $R(x, y, \lambda)$ obtained by Jakobson and Polterovich [2007] as well as estimates on averages of $R(x, y, \lambda)$ with respect to either $y \in M$ or $\lambda \in \mathbb{R}_{>0}$ studied by Lapointe, Polterovich and Safarov [Lapointe et al. 2009].

1.3. Discussion of Theorem 3. The scaling asymptotics (9) were first stated - without proof and without any assumptions on $\mathscr{L}_{x_{0}}$ - by Zelditch [2001, Theorem 2.1]. When $(M, g)=\left(S^{2}, g_{\text {round }}\right)$ is the standard 2-sphere, the square roots of the Laplace eigenvalues are $\lambda_{k}=k \cdot \sqrt{1+1 / k}$ for $k \in \mathbb{Z}_{+}$, and $\mathscr{L}_{x_{0}}=S_{x_{0}}^{*} M$, since the geodesic flow is $2 \pi$-periodic. There is therefore no $x_{0} \in S^{2}$ satisfying the assumptions of Theorem 3. Nonetheless, (8) holds with $E_{\lambda}$ replaced by the kernel of the spectral projection onto the $\lambda_{k}^{2}$ eigenspace and is known as Mehler-Heine asymptotics (see \$8.1 in [Szegó 1975]). More generally, on any Zoll manifold, the square roots of Laplace eigenvalues come in clusters that concentrate along an arithmetic progression. The width of the $k$-th cluster is on the order of $k^{-1}$ and we conjecture that the scaling asymptotics (8) hold for the spectral projectors onto these clusters (see [Zelditch 1997] for background on the spectrum of Zoll manifolds).

If one perturbs the standard metric on $S^{2}$ or on a Zoll surface, one can create smooth metrics possessing self-focal points $x_{0}$ where only a fraction of the measure of initial directions at $x_{0}$ give geodesics that return to $x_{0}$. These points complicate the remainder estimate for the general case. Indeed, it was pointed out to the authors by Safarov that even on the diagonal there is a two-term asymptotic formula with the second term of the form $Q(x, \lambda) \lambda^{n-1}$, where $Q$ is a bounded function. The function $Q$ is identically zero if $x_{0}$ is non-self-focal or if a full measure of geodesics emanating from $x_{0}$ return to $x_{0}$ at the same time. In general, however, $Q$ will contribute an extra term on the order of $\lambda^{n-1}$ to the asymptotics in (8). We refer the interested reader to $\S 1.8$ in [Safarov and Vassiliev 1997].

1.4. Notation. Given a Riemannian manifold $(M, g)$, let $\operatorname{vol}_{g}(M)$ be its volume, $\operatorname{dist}_{g}: M \times M \rightarrow \mathbb{R}$ be the induced distance function, and $\operatorname{inj}(M, g)$ be its injectivity radius. For $x \in M$ we write $S_{x}^{*} M$ for the unit sphere in the cotangent fiber $T_{x}^{*} M$. We denote by $\langle\cdot, \cdot\rangle_{g_{x}}: T_{x}^{*} M \times T_{x}^{*} M \rightarrow \mathbb{R}$ the Riemannian inner product on $T_{x}^{*} M$ and by $|\cdot|_{g_{x}}$ the corresponding norm. When $M=\mathbb{R}^{n}$ we simply write $\langle\cdot, \cdot\rangle$ and $|\cdot|$. In addition, for $(x, \xi) \in T^{*} M$, we will sometimes write $g_{x}^{1 / 2}(\xi)$ for the square root of the matrix $g_{x}$ applied to the covector $\xi$ and we write $\left|g_{x}\right|$ for the determinant of $g_{x}$.

We denote by $S^{k}$ the space of classical symbols of degree $k$, and we will write $S_{\text {hom }}^{k} \subseteq S^{k}$ for those symbols that are homogeneous of degree $k$. We also denote by $\Psi^{k}(M)$ the class of pseudodifferential operators of order $k$ on $M$.

\section{Outline for the proof of Theorem 2}

Fix $(M, g)$ and a non-self-focal point $x_{0} \in M$. Theorem 2 follows from the existence of a constant $c>0$ such that, for all $\varepsilon>0$, there exist $\tilde{\lambda}_{\varepsilon}>0$, an open neighborhood $U_{\varepsilon}$ of $x_{0}$ and a positive constant $c_{\varepsilon}$ such that

$$
\sup _{x, y \in U_{\varepsilon}}|R(x, y, \lambda)| \leq c \varepsilon \lambda^{n-1}+c_{\varepsilon} \lambda^{n-2}
$$


for all $\lambda \geq \tilde{\lambda}_{\varepsilon}$. Indeed, if $r_{\lambda}$ is a positive function with $\lim _{\lambda \rightarrow \infty} r_{\lambda}=0$, then it suffices to choose $\lambda_{\varepsilon}:=\max \left\{\tilde{\lambda}_{\varepsilon}, \inf \left\{\lambda: B\left(x_{0}, r_{\lambda}\right) \subset \mathcal{U}_{\varepsilon}\right\}\right\}$ to get

$$
\sup _{x, y \in B\left(x_{0}, r_{\lambda}\right)}|R(x, y, \lambda)| \leq c \varepsilon \lambda^{n-1}+c_{\varepsilon} \lambda^{n-2} \quad \text { for all } \lambda \geq \lambda_{\varepsilon} .
$$

By the definition of $R$ in (3) and the definition of $E_{\lambda}$, (2), we seek to find a constant $c>0$ such that, for all $\varepsilon>0$, there exist $\tilde{\lambda}_{\varepsilon}>0$, an open neighborhood $U_{\varepsilon}$ of $x_{0}$ and a positive constant $c_{\varepsilon}$ satisfying

$$
\sup _{x, y \in \mathscr{U}_{\varepsilon}}\left|E_{\lambda}(x, y)-\frac{\lambda^{n}}{(2 \pi)^{n}} \int_{|\xi|_{g_{y}}<1} e^{i \lambda\left\langle\exp _{y}^{-1}(x), \xi\right\rangle_{g_{y}}} \frac{d \xi}{\sqrt{\left|g_{y}\right|}}\right| \leq c \varepsilon \lambda^{n-1}+c_{\varepsilon} \lambda^{n-2}
$$

for all $\lambda \geq \tilde{\lambda}_{\varepsilon}$. We prove (17) using the so-called wave kernel method. That is, we use that the derivative of the spectral function is the inverse Fourier transform of the fundamental solution of the half-wave equation on $(M, g)$ :

$$
E_{\lambda}(x, y)=\int_{0}^{\lambda} \sum_{j} \delta\left(\mu-\lambda_{j}\right) \varphi_{j}(x) \varphi_{j}(y) d \mu=\int_{0}^{\lambda} \mathscr{F}_{t \rightarrow \mu}^{-1}(U(t, x, y))(\mu) d \mu,
$$

where $\mathscr{F}^{-1}$ denotes the inverse Fourier transform and $U(t, x, y)$ is the Schwartz kernel of $e^{-i t \sqrt{\Delta_{g}}}$. The singularities of $U(t, x, y)$ control the $\lambda \rightarrow \infty$ behavior of $E_{\lambda}$. We first study the contribution of the singularity of $U(t, x, y)$ coming at $t=\operatorname{dist}_{g}(x, y)$ by taking a Schwartz function $\rho \in \mathscr{Y}(\mathbb{R})$ that satisfies $\operatorname{supp}(\hat{\rho}) \subseteq(-\operatorname{inj}(M, g), \operatorname{inj}(M, g))$ and

$$
\hat{\rho}(t)=1 \quad \text { for all }|t|<\frac{1}{2} \operatorname{inj}(M, g) .
$$

We prove in Section 5 the following proposition, which shows that (17) holds with $E_{\lambda}$ replaced by $\rho * E_{\lambda}$.

Proposition 10 (smoothed projector). Let $(M, g)$ be a compact, smooth, Riemannian manifold of dimension $n \geq 2$ with no boundary. Then there exist constants $c, C>0$ such that

$$
\left|\rho * E_{\lambda}(x, y)-\frac{1}{(2 \pi)^{n}} \int_{|\xi|_{g_{y}}<\lambda} e^{\left.i \exp _{y}^{-1}(x), \xi\right\rangle_{g_{y}}} \frac{d \xi}{\sqrt{\left|g_{y}\right|}}\right| \leq c \operatorname{dist}_{g}(x, y) \lambda^{n-1}+C \lambda^{n-2}
$$

for all $x, y \in M$ with $\operatorname{dist}_{g}(x, y) \leq \frac{1}{2} \operatorname{inj}(M, g)$ and all $\lambda>0$.

Note that Proposition 10 does not assume that $x$ and $y$ are near a non-self-focal point. The reason is that convolving $E_{\lambda}$ with $\rho$ multiplies the half-wave kernel $U(t, x, y)$ in (18) by the Fourier transform $\hat{\rho}(t)$, which cuts out all but the singularity at $t=\operatorname{dist}_{g}(x, y)$. The proof of (20) relies on the construction in Section 3 of a short-time parametrix for $U(t)$, which differs from the celebrated Hörmander parametrix because it uses the coordinate-independent phase function

$$
\phi(t, x, y, \xi):=\left\langle\exp _{y}^{-1}(x), \xi\right\rangle_{g_{y}}-t|\xi|_{g_{y}}, \quad(t, x, y, \xi) \in \mathbb{R} \times M \times T^{*} M .
$$

It remains to estimate the difference $\left|E_{\lambda}(x, y)-\rho * E_{\lambda}(x, y)\right|$, which is the content of the following result: 
Proposition 11 (smooth vs. rough projector). Let $(M, g)$ be a compact, smooth, Riemannian manifold of dimension $n \geq 2$ with no boundary. Let $x_{0} \in M$ be a non-self-focal point. Then there exists $c>0$ such that, for all $\varepsilon>0$, there exist an open neighborhood $u_{\varepsilon}$ of $x_{0}$ and a positive constant $c_{\varepsilon}$ with

$$
\sup _{x, y \in \cup_{\varepsilon}}\left|E_{\lambda}(x, y)-\rho * E_{\lambda}(x, y)\right| \leq c \varepsilon \lambda^{n-1}+c_{\varepsilon} \lambda^{n-2}
$$

for all $\lambda \geq 1$.

The assumption that $x$ and $y$ are near a non-self-focal point $x_{0}$ guarantees that the dominant contribution to $E_{\lambda}(x, y)$ comes from the singularity of $U(t, x, y)$ at $t=\operatorname{dist}_{g}(x, y)$. Following the technique in [Sogge and Zelditch 2002], we prove Proposition 11 in Section 6 by microlocalizing $U(t)$ near $x_{0}$ (see Section 4) and applying two Tauberian-type theorems (presented in Section 6.1). Relation (17), and consequently Theorem 2, are a direct consequence of combining Proposition 10 with Proposition 11.

\section{Parametrix for the half-wave group}

The half-wave group is the one-parameter family of unitary operators $U(t)=e^{-i t \sqrt{\Delta_{g}}}$ acting on $L^{2}(M, g)$. It solves the initial value problem

$$
\left(\frac{1}{i} \partial_{t}+\sqrt{\Delta_{g}}\right) U(t)=0, \quad U(0)=\mathrm{Id},
$$

and its Schwartz kernel $U(t, x, y)$ is related to the kernel of the spectral projector $E_{\lambda}(x, y)$ via (18). It is well known (see [Duistermaat and Guillemin 1975; Hörmander 1985b]) that $U$ is a FIO in $I^{-1 / 4}(\mathbb{R} \times M, M ; \Gamma)$ associated to the canonical relation

$$
\Gamma=\left\{\left.(t, \tau, x, \eta, y, \xi) \in T^{*}(\mathbb{R} \times M \times M)|\tau=-| \xi\right|_{g_{y}}, G^{t}(y, \xi)=(x, \eta)\right\}
$$

where $G^{t}$ denotes geodesic flow.

Our goal in this section is to construct a short-time parametrix for $U(t)$ that is similar to Hörmander's parametrix [1968; 1985b, §29] but uses the coordinate-independent phase function $\phi: \mathbb{R} \times M \times T^{*} M \rightarrow \mathbb{R}$ defined in (21). Such a parametrix was used by Zelditch [2009], where a detailed construction was omitted. To construct the amplitude we follow [Laptev et al. 1994], who give a detailed treatment of FIOs that are built using global phase functions such as $\phi$. Denote by $\chi \in C^{\infty}([0,+\infty),[0,1])$ a compactly supported, smooth cut-off function with

$$
\operatorname{supp} \chi \subset[0, \operatorname{inj}(M, g)) \quad \text { and } \quad \chi(s)=1 \quad \text { for } s \in\left[0, \frac{1}{2} \operatorname{inj}(M, g)\right) \text {. }
$$

Further, following [Bérard et al. 1994; Berger et al. 1971, Proposition C.III.2], define

$$
\Theta(x, y):=\left|\operatorname{det}_{g} D_{\exp _{x}^{-1}(y)} \exp _{x}\right| \text {. }
$$

The subscript $g$ means that we use the inner products on $T_{\exp _{x}^{-1}(y)}\left(T_{x} M\right)$ and $T_{y}^{*} M$ induced from $g$ and, as explained in [Berger et al. 1971], $\Theta(x, y)=\sqrt{\left|g_{x}\right|}$ in normal coordinates at $y$. The main result of this section is the following: 
Proposition 12. For $|t|<\operatorname{inj}(M, g)$ we have

$$
U(t, x, y)=\frac{\chi\left(\operatorname{dist}_{g}(x, y)\right)}{(2 \pi)^{n} \Theta(x, y)^{1 / 2}} \int_{T_{y}^{*} M} e^{i \phi(t, x, y, \xi)} A(t, y, \xi) \frac{d \xi}{\sqrt{\left|g_{y}\right|}},
$$

where the equality is modulo smoothing kernels. The amplitude A, which is an order-0 polyhomogeneous symbol, is uniquely determined by $\phi$ modulo $S^{-\infty}$ and satisfies:

- For all $y \in M$ and $\xi \in T_{y}^{*} M$,

$$
A(0, y, \xi)=1
$$

- For $|t|<\operatorname{inj}(M, g)$ and all $(y, \xi) \in T_{y}^{*} M$, we have

$$
A(t, y, \xi)-1 \in S^{-1} \text {. }
$$

There are many choices of amplitude functions in (25) that depend on $t, x, y$ and $\xi$. When we write that $A$ is uniquely determined modulo $S^{-\infty}$, we mean that it is unique among amplitudes that are independent of $x$. The proof of Proposition 12 is divided into two steps. First, we prove in Section 3.1 that $\phi$ parametrizes $\Gamma$. Then, in Section 3.2, we construct the amplitude $A$.

3.1. Properties of the phase function. Throughout this section, we will denote by $\mathscr{T}_{y \rightarrow x}: T_{y}^{*} M \rightarrow T_{x}^{*} M$ the parallel transport operator (along the unique shortest geodesic from $x$ to $y$ ) for all $x$ and $y$ sufficiently close. We will use that

$$
\mathscr{T}_{y \rightarrow x} \exp _{y}^{-1}(x)=-\exp _{x}^{-1}(y) \quad \text { and } \quad \mathscr{T}_{y \rightarrow x}=\mathscr{T}_{x \rightarrow y}^{*} .
$$

Lemma 13. The phase function $\phi(t, x, y, \xi)$ parametrizes the canonical relation $\Gamma$ for $|t|<\operatorname{inj}(M, g)$ and $\operatorname{dist}_{g}(x, y)<\frac{1}{2} \operatorname{inj}(M, g)$, in the sense that

$$
\Gamma=i_{\phi}\left(C_{\phi}\right)
$$

is the image of the critical set

$$
C_{\phi}=\left\{(t, x, y, \xi) \in \mathbb{R} \times M \times T^{*} M \mid x=\exp _{y}\left(\frac{t \xi}{|\xi|_{g_{y}}}\right)\right\}
$$

under the immersion $i_{\phi}(t, x, y, \xi)=\left(t, d_{t} \phi, x, d_{x} \phi, y,-d_{y} \phi\right)$.

Proof. When $|t|<\operatorname{inj}(M, g)$, we have that $(t, x, y, \xi) \in C_{\phi}$ if and only if $t=0$ and $x=y$, or

$$
t=\operatorname{dist}_{g}(x, y) \neq 0 \text { and } \frac{\xi}{|\xi|_{g_{y}}}=\frac{\exp _{y}^{-1}(x)}{\operatorname{dist}_{g}(x, y)} .
$$

To prove (29) when $t=0$, we must show that

$$
i_{\phi}(0, x, x, \xi)=\left\{\left(0,-|\xi|_{g_{x}}, x, \xi, x, \xi\right) \mid \xi \in T_{x}^{*} M\right\}=\left.\Gamma\right|_{t=0}
$$

Since $\left.d_{x}\right|_{x=y} \exp _{y}^{-1}(x)$ is the identity on $T_{y}^{*} M$,

$$
\left.d_{x}\right|_{x=y} \phi(0, x, y, \xi)=\xi .
$$


Next, using (28), we have

$$
\phi(0, x, y, \xi)=\left\langle-\exp _{x}^{-1}(y), \mathscr{T}_{y \rightarrow x} \xi\right\rangle_{g_{x}}
$$

Therefore,

$$
\left.d_{y}\right|_{y=x} \phi(0, x, y, \xi)=-\xi
$$

which proves (30). To establish (29) when $t \neq 0$, we write

$$
\partial_{x_{k}} \phi(t, x, y, \xi)=\sum_{i, j} g^{i j}(y) \partial_{x_{k}}\left[\exp _{y}^{-1}(x)\right]_{i} \xi_{j}, \quad k=1, \ldots, n .
$$

Since $d_{x} \operatorname{dist}_{g}(x, y)=-\exp _{x}^{-1}(y) / \operatorname{dist}_{g}(x, y)$, evaluating (31) at

we obtain

$$
\xi=|\xi|_{g_{y}} \frac{\exp _{y}^{-1}(x)}{\operatorname{dist}_{g}(x, y)}
$$

$$
d_{x} \phi(t, x, y, \xi)=\frac{|\xi|_{g_{y}}}{2 \operatorname{dist}_{g}(x, y)} d_{x}\left[\operatorname{dist}_{g}(x, y)^{2}\right]=|\xi|_{g_{y}} d_{x} \operatorname{dist}_{g}(x, y)=-|\xi|_{g_{y}} \frac{\exp _{x}^{-1}(y)}{\operatorname{dist}_{g}(x, y)}
$$

Since $G^{t}\left(y, \exp _{y}^{-1}(x)\right)=\left(x,-\exp _{x}^{-1}(y)\right)$, it remains to check that

$$
-d_{y} \phi(t, x, y, \xi)=|\xi|_{y} \frac{\exp _{y}^{-1}(x)}{\operatorname{dist}_{g}(x, y)}
$$

which we verify in normal coordinates at $y$. We have that

$$
\left.d_{z}\right|_{z=y}|\xi|_{z}=0 \quad \text { and }\left.\quad \partial_{z_{k}}\right|_{z=y}\left(\exp _{z}^{-1}(x)\right)_{j}=-\delta_{k j}
$$

Thus,

$$
\left.\partial_{z_{k}}\right|_{z=y} \phi(t, x, z, \xi)=-\xi_{k} .
$$

Evaluating at $\xi=|\xi| \cdot x /|x|$, we find that

$$
-d_{y} \phi(t, x, y, \xi)=|\xi| \cdot \frac{x}{|x|}=|\xi|_{g_{y}} \frac{\exp _{y}^{-1}(x)}{\operatorname{dist}_{g}(x, y)}
$$

as desired.

We need one more lemma before constructing the amplitude $A$ in Proposition 12.

Lemma 14. Let $\beta: M \times M \rightarrow \mathbb{R}$ be any smooth function such that $\beta(x, x)=1$. The kernel of the identity operator acting on functions relative to the Riemannian volume form $\sqrt{\left|g_{y}\right|} d y$ admits the following representation as an oscillatory integral:

$$
\begin{aligned}
\delta(x, y) & =\frac{\chi\left(\operatorname{dist}_{g}(x, y)\right)}{(2 \pi)^{n}} \beta(x, y) \int_{T_{x}^{*} M} e^{-i\left\langle\exp _{x}^{-1}(y), \eta\right\rangle_{g_{x}}} \frac{d \eta}{\sqrt{\left|g_{x}\right|}} \\
& =\frac{\chi\left(\operatorname{dist}_{g}(x, y)\right)}{(2 \pi)^{n}} \beta(x, y) \int_{T_{y}^{*} M} e^{i\left\langle\exp _{y}^{-1}(x), \xi\right\rangle_{g_{y}}} \frac{d \xi}{\sqrt{\left|g_{y}\right|}} .
\end{aligned}
$$


Proof. Fix $x \in M$ and let $f \in C^{\infty}(M)$. Without loss of generality, assume that $f$ is supported in an open set $U \subset B(x, \operatorname{inj}(M, g))$ that contains the point $x$. Set $V=\exp _{x}^{-1}(U) \subset \mathbb{R}^{n}$ and consider normal coordinates at $x$ :

$$
h: V \rightarrow U, \quad h(z)=\exp _{x}(z)
$$

The pairing of the right-hand side of (33) with $f$ is then

$$
\frac{1}{(2 \pi)^{n}} \int_{\mathbb{R}^{n}} \int_{\mathbb{R}^{n}} e^{-i\langle z, \eta\rangle} \chi(|z|) f(h(z)) \beta(0, z) \sqrt{\left|g_{h(z)}\right|} d z d \eta=\chi(|0|) f(h(0)) \sqrt{\left|g_{h(0)}\right|} \beta(0,0)=f(x) .
$$

This proves (33). To explain why the two oscillatory integrals in the statement of the present lemma define the same distribution, we will use the parallel transport operator (see (28)). We write (33) as

$$
\frac{\chi\left(\operatorname{dist}_{g}(x, y)\right)}{(2 \pi)^{n}} \beta(x, y) \int_{T_{x}^{*} M} e^{i\left\langle\exp _{y}^{-1}(x), \mathcal{T}_{y \rightarrow x} \eta\right\rangle_{g y}} \frac{d \eta}{\sqrt{\left|g_{x}\right|}} .
$$

Let $\left(y^{1}, \ldots, y^{n}\right)$ be any local coordinates near $x$. We note that, for every $y$, the collection of covectors $\left\{\left.g_{y}^{1 / 2} d y^{j}\right|_{y}\right\}_{j=1}^{n}$ is an orthonormal basis for $T_{y}^{*} M$. Hence, the Lebesgue measure on $T_{y}^{*} M$ in our coordinates is $\left.\left.\left|g_{y}\right|^{1 / 2} d y^{1}\right|_{y} \wedge \cdots \wedge d y^{n}\right|_{y}$ and, since $\mathscr{T}_{y \rightarrow x}$ is an isometry,

$$
\xi=\mathscr{T}_{y \rightarrow x} \eta \quad \Longrightarrow \quad d \xi=\frac{\left|g_{y}\right|^{1 / 2}}{\left|g_{x}\right|^{1 / 2}} d \eta
$$

This allows us to change variables in (35) to obtain the integral over $T_{y}^{*} M$ in the statement of the lemma.

3.2. Construction of the amplitude. To construct the amplitude $A$ in Proposition 12 , let us write $\tilde{U}(t)$ for the wave operator acting on sections of the half-density bundle $\Omega^{1 / 2}(M)$. Lemma 13 combined with Theorem 3.4 in [Laptev et al. 1994] (or Proposition 25.1.5 in [Hörmander 1985b]) shows that there exists a polyhomogeneous symbol $A$ of order 0 that is supported in a neighborhood of $C_{\phi}$ for which

$$
\tilde{U}(t, x, y)=\frac{\chi\left(\operatorname{dist}_{g}(x, y)\right)}{(2 \pi)^{n}} \int_{T_{y}^{*} M} e^{i \phi(t, x, y, \xi)} A(t, y, \xi) d_{\phi}(t, x, y, \xi) d \xi \quad\left(\bmod C^{\infty}\right),
$$

where

$$
d_{\phi}=\sqrt{\left|\operatorname{det} d_{x, \xi} \phi\right|} \in \Omega_{x}^{1 / 2}(M) \otimes \Omega_{y}^{-1 / 2}(M)
$$

is a $\frac{1}{2}$-density in $x$ and a $\left(-\frac{1}{2}\right)$-density in $y$. Since $d \xi$ behaves like a 1-density in $y, \tilde{U}(t, x, y)$ is in $\Omega_{x}^{1 / 2}(M) \otimes \Omega_{y}^{1 / 2}(M)$. The square root of the Riemannian volume form,

$$
g_{y}^{1 / 4}=\left|g_{y}\right|^{1 / 4}|d y|^{1 / 2} \in \Omega_{y}^{1 / 2}(M),
$$

identifies $L^{2}$ global sections $\Gamma\left(\Omega^{1 / 2}(M)\right)$ with $L^{2}(M)$ via

$$
L^{2}(M) \rightarrow \Gamma\left(\Omega^{1 / 2}(M)\right), \quad f(y) \mapsto f(y) \cdot g_{y}^{1 / 4} .
$$


Then, computing in normal coordinates at $y$, we have

$$
d_{\phi}(t, x, y, \xi) g_{y}^{1 / 4} g_{x}^{-1 / 4}=\frac{1}{\left|g_{x}\right|^{1 / 4}}=\frac{1}{\Theta(x, y)^{1 / 2}} .
$$

In addition, since $U(t, x, y)=\tilde{U}(t, x, y) g_{x}^{-1 / 4} g_{y}^{-1 / 4}$, relation (37) gives

$$
U(t, x, y)=\frac{\chi\left(\operatorname{dist}_{g}(x, y)\right)}{(2 \pi)^{n} \Theta(x, y)^{1 / 2}} \int_{T_{y}^{*} M} e^{i \phi(t, x, y, \xi)} A(t, y, \xi) \frac{d \xi}{\sqrt{\left|g_{y}\right|}} \quad\left(\bmod C^{\infty}\right) .
$$

Write $A \sim \sum_{j \geq 0} A_{-j}$ for the polyhomogeneous expansion of $A$. Note that

$$
A_{0}(t, y, \xi)=1 \text { for all } t,
$$

because the principal symbol $\tilde{U}(t)$ is independent of $t$ and equals 1 at $t=0$ [Laptev et al. 1994, Theorem 4.1]. Next, since

$$
\tilde{U}(0, x, y)=\frac{\chi\left(\operatorname{dist}_{g}(x, y)\right)}{(2 \pi)^{n}} \int_{T_{y}^{*} M} e^{i \phi(t, x, y, \xi)} A(0, y, \xi) d_{\phi}(t, x, y, \xi) \frac{d \xi}{\sqrt{\left|g_{y}\right|}}
$$

is a kernel for the identity modulo $C^{\infty}$ and $A(0, y, \xi)$ is uniquely determined by $\phi$ mod $S^{-\infty}$ (Theorem 3.4 in [Laptev et al. 1994]), it follows from Lemma 14 and (37), with $\beta(x, y)=\Theta(x, y)^{-1 / 2}$, that

$$
A_{-j}(0, y, \xi)=0 \text { for all } j \geq 1,
$$

as desired.

\section{Microlocalizing the identity operator at non-self-focal points}

In this section we microlocalize the identity operator near a non-self-focal point $x_{0}$. For every $\varepsilon>0$ we make a microlocal decomposition of the identity, $\mathrm{Id}=B_{\varepsilon}+C_{\varepsilon}$ near $x_{0}$, where the operator $B_{\varepsilon}$ is supported on the set of "bad" loopset directions and is built so that its support has measure smaller than $\varepsilon$. This construction follows closely that of Sogge and Zelditch [2002].

Lemma 15. There exists a constant $\gamma>0$ such that, for every $\varepsilon>0$, there is a neighborhood $O_{\varepsilon}$ of $x_{0}$, a function $\psi_{\varepsilon} \in C_{c}^{\infty}(M)$ and real-valued operators $B_{\varepsilon}, C_{\varepsilon} \in \Psi^{0}(M)$ supported in $\mathbb{O}_{\varepsilon}$ satisfying the following properties:

(1) For every $\varepsilon, \operatorname{supp}\left(\psi_{\varepsilon}\right) \subset \mathfrak{O}_{\varepsilon}$ and $\psi_{\varepsilon}=1$ on a neighborhood of $x_{0}$.

(2) For every $\varepsilon$,

$$
B_{\varepsilon}+C_{\varepsilon}=\psi_{\varepsilon}^{2} .
$$

(3) $U(t) C_{\varepsilon}^{*}$ is a smoothing operator for $\frac{1}{2} \operatorname{inj}(M, g)<|t|<\frac{1}{\varepsilon}$.

(4) Denote by $b_{0}$ and $c_{0}$ the principal symbols of $B_{\varepsilon}$ and $C_{\varepsilon}$ respectively. Then, for all $x \in M$, we have

$$
\frac{1}{\varepsilon} \int_{|\xi|_{g_{x}} \leq 1}\left|b_{0}(x, \xi)\right|^{2} d \xi+\int_{|\xi|_{g_{x}} \leq 1}\left|c_{0}(x, \xi)\right|^{2} d \xi \leq \gamma
$$

and both $b_{0}$ and $c_{0}$ are constant in an open neighborhood of $x_{0}$. 
Proof. For every $x, y \in M$ and $\xi \in S_{x}^{*} M$, define the loopset function

$$
\mathscr{L}^{*}(x, y, \xi)=\inf \left\{t>0 \mid \exp _{x}(t \xi)=y\right\}
$$

with $\mathscr{L}^{*}(x, y, \xi)=+\infty$ if the infimum is taken over the empty set. Unlike the loopset function studied in [Sogge and Zelditch 2002], we are interested in $x \neq y$ (but with $\operatorname{dist}_{g}(x, y)<\frac{1}{2} \operatorname{inj}(M, g)$ ).

Fix a coordinate chart $\left(\kappa_{x_{0}}, \mathscr{V}_{x_{0}}\right)$ containing $x_{0}$ with $\kappa_{x_{0}}: \mathscr{V}_{x_{0}} \subset \mathbb{R}^{n} \rightarrow M$. We first note that the function $f: \mathscr{V}_{x_{0}} \times \mathscr{V}_{x_{0}} \times S^{n-1} \rightarrow \mathbb{R}$ defined as $f(x, y, \xi)=1 / \mathscr{L}^{*}(x, y, \xi)$ is upper semicontinuous and so, by the proof of [Sogge and Zelditch 2002, Lemma 3.1], there exist a neighborhood $\mathcal{N}_{\varepsilon} \subset \mathscr{V}_{x_{0}}$ of $x_{0}$ and an open set $\Omega_{\varepsilon} \subset S^{n-1}$ for which

$$
\begin{aligned}
\mathscr{L}^{*}(x, y, \xi) & >\frac{1}{\varepsilon} \quad \text { in } \mathcal{N}_{\varepsilon} \times \mathcal{N}_{\varepsilon} \times \Omega_{\varepsilon}^{c}, \\
\left|\Omega_{\varepsilon}\right| & \leq \varepsilon .
\end{aligned}
$$

In addition, there exists a function $\varrho_{\varepsilon} \in C^{\infty}\left(S^{n-1},[0,1]\right)$ satisfying that $\varrho_{\varepsilon} \equiv 1$ on $\Omega_{\varepsilon}, \varrho_{\varepsilon}(\xi)=\varrho(-\xi)$ for all $\xi \in S^{n-1}$ and $\left|\operatorname{supp}\left(\varrho_{\varepsilon}\right)\right|<2 \varepsilon$. In particular,

$$
\mathscr{L}^{*}(x, y, \xi)>\frac{1}{\varepsilon} \quad \text { on } \mathcal{N}_{\varepsilon} \times \mathcal{N}_{\varepsilon} \times \operatorname{supp}\left(1-\varrho_{\varepsilon}\right) .
$$

As in [Sogge and Zelditch 2002], we choose a real-valued function $\tilde{\psi}_{\varepsilon} \in C_{c}^{\infty}\left(\mathbb{R}^{n}\right)$ with $\operatorname{supp}\left(\tilde{\psi}_{\varepsilon}\right) \subset \mathcal{N}_{\varepsilon}$ that is equal to 1 in a neighborhood of $\kappa_{x_{0}}^{-1}\left(x_{0}\right)$. Define symbols on $\mathbb{R}^{3 n}$ by

$$
\tilde{b}_{\varepsilon}(x, y, \xi)=\tilde{\psi}_{\varepsilon}(x) \tilde{\psi}_{\varepsilon}(y) \varrho_{\varepsilon}\left(\frac{\xi}{|\xi|}\right) \quad \text { and } \quad \tilde{c}_{\varepsilon}(x, y, \xi)=\tilde{\psi}_{\varepsilon}(x) \tilde{\psi}_{\varepsilon}(y)\left(1-\varrho_{\varepsilon}\left(\frac{\xi}{|\xi|}\right)\right),
$$

and consider their respective quantizations $\operatorname{Op}\left(\tilde{b}_{\varepsilon}\right), \operatorname{Op}\left(\tilde{c}_{\varepsilon}\right) \in \Psi^{0}\left(\mathbb{R}^{n}\right)$. Properties (1) and (2) follow from setting

$$
B_{\varepsilon}:=\left(\kappa_{x_{0}}^{-1}\right)^{*} \operatorname{Op}\left(\tilde{b}_{\varepsilon}\right), \quad C_{\varepsilon}:=\left(\kappa_{x_{0}}^{-1}\right)^{*} \operatorname{Op}\left(\tilde{c}_{\varepsilon}\right)
$$

and

$$
\mathrm{O}_{\varepsilon}=\kappa_{x_{0}}\left(\mathcal{N}_{\varepsilon}\right), \quad \psi_{\varepsilon}:=\left(\kappa_{x_{0}}^{-1}\right)^{*} \tilde{\psi}_{\varepsilon} .
$$

Note that if, for some time, $\frac{1}{2} \operatorname{inj}(M, g)<t<\frac{1}{\varepsilon}$, we have $\exp _{x}(t \xi /|\xi|)=y$ for some $x, y \in M$ and $\xi \in T_{x}^{*} M$, then $\mathscr{L}^{*}(x, y, \xi /|\xi|) \leq \frac{1}{\varepsilon}$, and the latter implies $\tilde{c}_{\varepsilon}(x, y, \xi)=0$. Therefore, we see that, if we write $c_{\varepsilon}$ for the symbol of $C_{\varepsilon}$, then

$$
c_{\varepsilon}(x, y, \xi)=0 \quad \text { if } \quad(t, x, y ; \tau, \xi, \eta) \in \Gamma \quad \text { with } \quad \frac{1}{2} \operatorname{inj}(M, g)<t<\frac{1}{\varepsilon},
$$

where $\Gamma$ is the canonical relation underlying $U(t)$ (see (23)). Thus, the kernel of $U(t) C_{\varepsilon}^{*}$ is a smooth function for $\frac{1}{2} \operatorname{inj}(M, g)<t<\frac{1}{\varepsilon}$ and for $(x, y)$ in $\mathbb{O}_{\varepsilon} \times \mathbb{O}_{\varepsilon}$, which is precisely statement (3). For all $x \in \mathcal{N}_{\varepsilon}$, we have that the principal symbols $b_{0}$ and $c_{0}$ satisfy the inequality (40), since $\left|\operatorname{supp} \varrho_{\varepsilon}\right|<2 \varepsilon$. Also, since $b_{\varepsilon}$ and $c_{\varepsilon}$ are real valued and invariant under $\xi \mapsto-\xi$, we have that $B_{\varepsilon}$ and $C_{\varepsilon}$ are real valued as well. 
Remark 16. By construction, the subprincipal symbols of $B_{\varepsilon}$ and $C_{\varepsilon}$ (acting on half-densities) are zero in a neighborhood of $x_{0}$. Indeed, the principal symbols are constant as functions of $x$ in a neighborhood of $x_{0}$ and, in the coordinates $\kappa_{x_{0}}$ used in Lemma 15, the total symbols of $B_{\varepsilon}$ and $C_{\varepsilon}$ are homogeneous functions of order zero. Thus, in any coordinates, the parts of order -1 of the polyhomogeneous expansions of the total symbols of $B_{\varepsilon}$ and $C_{\varepsilon}$ vanish in a neighborhood of $x_{0}$.

Remark 17. We record precise asymptotics for the on-diagonal behavior of $Q E Q^{*}(x, x, \mu)$ for all $x \in \mathscr{O}_{\varepsilon}$ and $Q \in\left\{\operatorname{Id}, B_{\varepsilon}, C_{\varepsilon}\right\}$. Write $q_{0}$ for the principal symbol of $Q$. Using that the subprincipal symbols of both $Q$ and $Q Q^{*}$ (acting on half-densities) vanish identically in a neighborhood $\widetilde{O}_{\varepsilon}$ of $x_{0}$, Lemmas 3.2 and 3.3 in [Sogge and Zelditch 2002] show that there exist constants $c, c_{\varepsilon}>0$ such that, for all $x \in \widetilde{O}_{\varepsilon}$,

$$
\operatorname{QEQ}^{*}(x, x, \lambda)=\frac{1}{(2 \pi)^{n}} \int_{|\xi|_{g_{x}}<\lambda}\left|q_{0}(x, \xi)\right|^{2} d \xi+R_{Q}(x, x, \lambda)
$$

with

$$
\left|R_{Q}(x, x, \lambda)\right| \leq c \varepsilon \lambda^{n-1}+c_{\varepsilon} \lambda^{n-2}
$$

for all $\lambda \geq 1$. We note that a similar result is obtained in [Safarov and Vassiliev 1997, Theorem 1.8.7], with the difference that the latter is proved for points $x$ that are nonfocal.

\section{Smoothed projector: proof of Proposition 10}

Proposition 18 below is our main technical estimate on $E_{\lambda}(x, y)$. We use it to prove Propositions 10 and 11 in Sections 5 and 6, respectively.

Proposition 18. Let $(M, g)$ be a compact, smooth, Riemannian manifold of dimension $n \geq 2$ with no boundary. Let $\varepsilon>0$ and $Q \in\left\{\operatorname{Id}, B_{\varepsilon}, C_{\varepsilon}\right\}$ for $B_{\varepsilon}$ and $C_{\varepsilon}$, as introduced in Lemma 15. Let $q_{0}$ be the principal symbol of $Q$. Then, for all $x, y \in O_{\varepsilon}$ with $\operatorname{dist}_{g}(x, y) \leq \frac{1}{2} \operatorname{inj}(M, g)$ and all $\mu \geq 1$, we have

$$
\begin{aligned}
& \partial_{\mu}\left(\rho * E Q^{*}\right)(x, y, \mu) \\
& =\frac{\mu^{n-1}}{(2 \pi)^{n} \Theta(x, y)^{1 / 2}}\left[\int_{S_{y}^{*} M} e^{i\left\langle\exp _{y}^{-1}(x), \omega\right\rangle_{g_{y}}} q_{0}(y, \omega) \frac{d \omega}{\sqrt{\left|g_{y}\right|}}\right. \\
& \left.\quad+\int_{S_{y}^{*} M} e^{i\left\langle\exp _{y}^{-1}(x), \omega\right\rangle_{g_{y}}} D_{-1}^{Q}(y, \omega) \frac{d \omega}{\sqrt{\left|g_{y}\right|}}\right]+W(x, y, \mu) .
\end{aligned}
$$

Here, $d \omega$ is the Euclidean surface measure on $S_{y}^{*} M$ and the function $\Theta$ is as defined in (24). The function $D_{-1}^{Q}$ belongs to $S^{-1}$ and there exists $C>0$ such that, for every $\varepsilon>0$,

$$
\begin{gathered}
D_{-1}^{B_{\varepsilon}}(y, \xi)+D_{-1}^{C_{\varepsilon}}(y, \xi)=0 \text { for all } y \in \mathscr{O}_{\varepsilon}, \\
\sup _{x, y \in O_{\varepsilon}}\left|\int_{S_{y}^{*} M} e^{i\left\langle\exp _{y}^{-1}(x), \omega\right\rangle_{g_{y}}} D_{-1}^{Q}(y, \omega) \frac{d \omega}{\sqrt{\left|g_{y}\right|}}\right| \leq C \varepsilon .
\end{gathered}
$$

In addition, $W$ is a smooth function in $(x, y)$ for which there exists $C>0$ such that, for all $\mu>0$,

$$
\sup _{\operatorname{dist}_{g}(x, y) \leq \frac{1}{2} \operatorname{inj}(M, g)}|W(x, y, \mu)| \leq C\left(\mu^{n-2} \operatorname{dist}_{g}(x, y)+(1+\mu)^{n-3}\right) .
$$


Proof. Let $x, y \in M$ with $\operatorname{dist}_{g}(x, y) \leq \frac{1}{2} \operatorname{inj}(M, g)$. Note that

$$
\partial_{\mu}\left(\rho * E Q^{*}\right)(x, y, \mu)=\frac{1}{2 \pi} \int_{-\infty}^{+\infty} e^{i t \lambda} \hat{\rho}(t) U(t) Q^{*}(x, y) d t .
$$

We start by rewriting $U(t) Q^{*}(x, y)$ using the parametrix (25) for $U(t)$. We have

$$
U(t) Q^{*}(x, y)=\frac{\chi\left(d_{g}(x, y)\right)}{(2 \pi)^{n} \Theta(x, y)^{1 / 2}} \int_{T_{y}^{*} M} e^{i\left\langle\exp _{y}^{-1}(x), \xi\right\rangle_{g_{y}}-t|\xi|_{g_{y}}} D^{\left.Q_{(}, y, \xi\right)} \frac{d \xi}{\sqrt{\left|g_{y}\right|}}
$$

for some $D^{Q} \in S^{0}$ with polyhomogeneous expansion $D^{Q} \sim \sum_{j \geq 0} D_{-j}^{Q}$. We claim that

$$
D_{0}^{Q}(0, y, \xi)=q_{0}(y, \xi)
$$

and that, for all $\varepsilon>0$,

$$
\begin{aligned}
& D_{-1}^{B^{\varepsilon}}(0, y, \xi)+D_{-1}^{C^{\varepsilon}}(0, y, \xi)=0, \\
& \sup _{x, y \in \bigcirc_{\varepsilon}}\left|\int_{S_{y}^{*} M} e^{i\left(\exp _{y}^{-1}(x), \omega\right\rangle_{g y}} D_{-1}^{Q}(0, y, \xi) \frac{d \omega}{\sqrt{\left|g_{y}\right|}}\right| \leq C \varepsilon,
\end{aligned}
$$

where $C$ is a constant independent of $\varepsilon$. Indeed, let $\tilde{U}(t) \tilde{Q}^{*}$ denote the operator $U(t) Q^{*}$ when regarded as acting on half-densities and note that, by the same computations that deduce (38) from (36), we have

$$
\tilde{U}(t) \tilde{Q}^{*}(x, y)=\frac{\chi\left(d_{g}(x, y)\right)}{(2 \pi)^{n}} \int_{T_{y}^{*} M} e^{i\left\langle\exp _{y}^{-1}(x), \xi\right\rangle_{g y}-t|\xi|_{g_{y}}} D^{\left.Q_{(t, y}, \xi\right) d_{\phi}(t, x, y, \xi) d \xi}
$$

Since the principal symbols of both $\widetilde{U}$ and $\widetilde{Q}$ are independent of $t$, and $\tilde{U}(0)=\mathrm{Id}$, we know

$$
D_{0}^{Q}(t, y, \xi)=q_{0}(y, \xi) .
$$

Moreover, note that $D_{-1}^{\mathrm{Id}}(0, y, \xi)=0$ by Proposition 12 and that $D^{\mathrm{Id}}$ is uniquely determined modulo $S^{-\infty}$ by the phase function $\phi$ (see [Laptev et al. 1994]). This proves (51), since on $0_{\varepsilon}$ we have $\operatorname{Id}=B_{\varepsilon}+C_{\varepsilon}$. Finally, by the construction of $B_{\varepsilon}$, we see that the size of the support of $D_{-1}^{B_{\varepsilon}}(0, y, \xi)$ is smaller than a constant times $\varepsilon$. This proves (52) for $Q=B_{\varepsilon}$ and hence for $Q=C_{\varepsilon}$, since $D_{-1}^{B_{\varepsilon}}=-D_{-1}^{C_{\varepsilon}}$.

Combining (48) and (49) and changing coordinates $\xi \mapsto \mu r \omega$, where $(r, \omega) \in[0,+\infty) \times S_{y}^{*} M$, we obtain up to an $O\left(\mu^{-\infty}\right)$ error that

$$
\begin{aligned}
& \Theta(x, y)^{1 / 2} \cdot \partial_{\mu}\left(\rho * E Q^{*}\right)(x, y, \mu) \\
& \quad=\frac{\mu^{n}}{(2 \pi)^{n+1}} \int_{\mathbb{R}} \int_{0}^{\infty} \hat{\rho}(t) e^{i \mu t(1-r)} \chi(r) r^{n-1}\left(\int_{S_{y}^{*} M} e^{i \mu r\left\langle\exp _{y}^{-1}(x), \omega\right\rangle_{y}} D^{Q}(t, y, r \mu \omega) d \omega\right) d r d t,
\end{aligned}
$$

where $\chi \in C_{c}^{\infty}(\mathbb{R})$ is a cut-off function that is identically 1 near $r=1$ and vanishes for $r \notin\left[\frac{1}{2}, \frac{3}{2}\right]$. Indeed, on the support of $1-\chi$, the operator $L=(1 / i \mu(1-r)) \partial_{t}$ is well defined, preserves $e^{i \mu t(1-r)}$, and its adjoint $L^{*}$ satisfies that, for all $k \in \mathbb{Z}^{+}$,

$$
\left|\left(L^{*}\right)^{k}\left(r^{n-1}(1-\chi(r)) \hat{\rho}(t) \int_{S_{y}^{*} M} e^{i \mu r\left\langle\exp _{y}^{-1}(x), \omega\right\rangle_{y}} D^{Q}(t, y, r \mu \omega) d \omega\right)\right| \leq(1+\mu)^{-k} \cdot c_{k}
$$


for some $c_{k}>0$. Define

$$
S^{Q}(t, y, \xi):=q_{0}(y, \xi)+D_{-1}^{Q}(t, y, \xi)
$$

to be the two leading terms of $D^{Q}$. Since $D^{Q}-S^{Q} \in S^{-2}$, up to a $O\left(\mu^{n-3}\right)$ error we have

$$
\begin{aligned}
& \Theta(x, y)^{1 / 2} \cdot \partial_{\mu}\left(\rho * E Q^{*}\right)(x, y, \mu) \\
& \quad=\frac{\mu^{n}}{(2 \pi)^{n+1}} \int_{\mathbb{R}} \int_{0}^{\infty} \hat{\rho}(t) e^{i \mu t(1-r)} \chi(r) r^{n-1}\left(\int_{S_{y}^{*} M} e^{\left.i \mu r\left\langle\exp _{y}^{-1}(x), \omega\right\rangle_{g_{y}} S Q_{(t, y, r \mu \omega)} d \omega\right) d r d t .}\right.
\end{aligned}
$$

According to [Sogge 1993, Theorem 1.2.1], there exist smooth functions $a_{ \pm}, b_{ \pm} \in C^{\infty}\left(M \times \mathbb{R}^{n}\right)$ such that, for all $(y, \eta) \in M \times T_{y}^{*} M$,

$$
\int_{S_{y}^{*} M} e^{i\langle\eta, \omega\rangle_{g_{y}}} Q_{(t, y, \mu r \omega)} \frac{d \omega}{\sqrt{\left|g_{y}\right|}}=\sum_{ \pm} e^{ \pm i|\eta|_{g_{y}}}\left(a_{ \pm}(y, \eta)+r^{-1} \mu^{-1} \cdot b_{ \pm}(t, y, \eta)\right)
$$

and

$$
\begin{aligned}
\left|\partial_{\eta}^{\alpha} a_{ \pm}(y, \eta)\right| & \leq C_{\alpha}\left(1+|\eta| g_{y}\right)^{-(n-1) / 2-|\alpha|}, \\
\left|\partial_{t}^{\beta} \partial_{\eta}^{\alpha} b_{ \pm}(t, y, \eta)\right| & \leq C_{\alpha, \beta}\left(1+|\eta|_{g_{y}}\right)^{-(n-1) / 2-|\alpha|-1},
\end{aligned}
$$

for all multi-indices $\alpha \geq 0$ and $\beta \geq 0$ and for some $C_{\alpha}, C_{\alpha, \beta}>0$ independent of $t, y$ and $\eta$. Hence, (54) equals

$$
\frac{\mu^{n}}{(2 \pi)^{n+1}} \sum_{ \pm} \int_{\mathbb{R}} \int_{0}^{\infty} e^{i \mu \psi_{ \pm}(t, r, x, y)} g_{ \pm}(t, r, x, y, \mu) d r d t
$$

where $\psi_{ \pm}(t, r, x, y)=t(1-r) \pm r \operatorname{dist}_{g}(x, y)$ and

$$
g_{ \pm}(t, r, x, y, \mu)=\frac{1}{(2 \pi)^{n}} r^{n-1} \chi(r) \hat{\rho}(t)\left(a_{ \pm}\left(y, r \mu \exp _{y}^{-1}(x)\right)+r^{-1} \mu^{-1} b_{ \pm}\left(t, y, r \mu \exp _{y}^{-1}(x)\right)\right) .
$$

Note that the critical points of $\psi_{ \pm}$are $\left(t_{c}^{ \pm}, r_{c}^{ \pm}\right)=\left( \pm \operatorname{dist}_{g}(x, y), 1\right)$ and that

$$
\operatorname{det}\left(\operatorname{Hess} \psi_{ \pm}\left(t_{c}^{ \pm}, r_{c}^{ \pm}, x, y\right)\right)=1 .
$$

Hence, we apply the method of stationary phase to get that (58) is

$$
\begin{aligned}
\mu^{n-1} e^{ \pm i \mu \operatorname{dist}_{g}(x, y)} \sum_{ \pm}\left(g_{ \pm}\left(t_{c}^{ \pm}, r_{c}^{ \pm}, x, y, \mu\right)-i \mu^{-1} \partial_{r} \partial_{t} g_{ \pm}\left(t_{c}^{ \pm}, r_{c}^{ \pm}, x, y, \mu\right)\right) & \\
& +O\left(\mu^{n-3} \sup _{(t, r) \in \operatorname{supp}\left(g_{ \pm}\right)} \sup _{\alpha+\beta \leq 7}\left|\partial_{t}^{\alpha} \partial_{r}^{\beta} g_{ \pm}(t, r, x, y, \mu)\right|\right) .
\end{aligned}
$$

We take 7 derivatives in the last term, since, in stationary phase with a quadratic phase over $\mathbb{R}^{k}$, the remainder after the first $N$ terms is bounded by $k+1+2 N$ derivatives of the amplitude. Note that $\partial_{t} \hat{\rho}(t)=0$ for $t= \pm \operatorname{dist}_{g}(x, y)$. Hence, since $a_{ \pm}$are independent of $t$, we have

$$
i \mu^{-1} \partial_{r} \partial_{t} g_{ \pm}\left(t_{c}^{ \pm}, r_{c}^{ \pm}, x, y, \mu\right)=O\left(\mu^{-2}\right) .
$$


Moreover, by (56) and (57), the derivatives of $g$ in $t$ and $r$ are uniformly bounded. Hence,

$$
\frac{\mu^{n-1}}{(2 \pi)^{n}} \int_{S_{y}^{*} M} e^{i\left\langle\exp _{y}^{-1}(x), \omega\right\rangle_{g_{y}}}\left(q_{0}(y, \omega)+\mu^{-1} D_{-1}^{Q}\left(\operatorname{dist}_{g}(x, y), y, \omega\right)\right) \frac{d \omega}{\sqrt{\left|g_{y}\right|}}+O\left(\mu^{n-3}\right) .
$$

Taylor-expanding $D_{-1}^{Q}\left(\operatorname{dist}_{g}(x, y), y, \omega\right)=D_{-1}^{Q}(0, y, \omega)+O\left(\operatorname{dist}_{g}(x, y)\right)$ and recalling (51) and (52) completes the proof.

Proof of Proposition 10. Proposition 10 follows by integrating (44) with respect to $\mu$ from 0 to $\lambda$ applied to $Q=$ Id. We have

$$
\rho * E(x, y, \lambda)=\int_{0}^{\lambda} \frac{\mu^{n-1}}{(2 \pi)^{n} \Theta(x, y)^{1 / 2}}\left(\int_{S_{y}^{*} M} e^{i \mu\left\langle\exp _{y}^{-1}(x), \omega\right\rangle_{y}} \frac{d \omega}{\sqrt{\left|g_{y}\right|}}\right) d \mu+\int_{0}^{\lambda} W(x, y, \mu) d \mu .
$$

Changing coordinates to $\xi=\mu \omega$, we find

$$
\rho * E(x, y, \lambda)=\frac{\lambda^{n}}{(2 \pi)^{n} \Theta(x, y)^{1 / 2}} \int_{|\xi|_{g_{y}}<1} e^{i \lambda\left\langle\exp _{y}^{-1}(x), \xi\right\rangle_{g_{y}}} \frac{d \xi}{\sqrt{\left|g_{y}\right|}}+\int_{0}^{\lambda} W(x, y, \mu) d \mu .
$$

Note that

$$
\Theta(x, y)^{-1 / 2}=1+O\left(\operatorname{dist}_{g}(x, y)^{2}\right)
$$

and

$$
\frac{\exp _{y}^{-1}(x)}{i \lambda \operatorname{dist}_{g}(x, y)^{2}} \nabla_{\xi} e^{i \lambda\left\langle\exp _{y}^{-1}(x), \xi\right\rangle_{y}}=e^{i \lambda\left\langle\exp _{y}^{-1}(x), \xi\right\rangle_{g_{y}}}
$$

Therefore, we may integrate by parts once in (64) to obtain

$$
\begin{aligned}
\rho * E(x, y, \lambda)=\frac{\lambda^{n}}{(2 \pi)^{n}} \int_{|\xi|_{y y}<1} e^{i \lambda\left\langle\exp _{y}^{-1}(x), \xi\right\rangle_{g}} \frac{d \xi}{\sqrt{\left|g_{y}\right|}}+\int_{0}^{\lambda} W(x, y, \mu) d \mu & \\
& +O\left(\operatorname{dist}_{g}(x, y) \lambda^{n-1} \int_{|\xi|_{g_{y}}=1} e^{i \lambda\left\langle\exp _{y}^{-1}(x), \omega\right\rangle_{g_{y}}} d \omega\right) .
\end{aligned}
$$

Since

$$
\sup _{\operatorname{dist} g(x, y)<\operatorname{inj}(M, g)}\left|\operatorname{dist}_{g}(x, y) \int_{|\xi|_{g_{y}}=1} e^{i \lambda\left\langle\exp _{y}^{-1}(x), \omega\right\rangle_{g_{y}}} d \omega\right|=o(1)
$$

as $\lambda \rightarrow \infty$, we find that

$$
\rho * E(x, y, \lambda)=\frac{\lambda^{n}}{(2 \pi)^{n}} \int_{|\xi|_{g y}<1} e^{i \lambda\left\langle\exp _{y}^{-1}(x), \xi\right\rangle_{g_{y}}} \frac{d \xi}{\sqrt{\left|g_{y}\right|}}+\int_{0}^{\lambda} W(x, y, \mu) d \mu+o\left(\lambda^{n-1}\right) .
$$

By (47), we have

$$
\sup _{x, y \in B\left(x_{0}, \operatorname{inj}(M, g) / 2\right)}\left|\int_{0}^{\lambda} W(x, y, \mu) d \mu\right| \leq c \operatorname{dist}_{g}(x, y) \lambda^{n-1}+C \lambda^{n-2}
$$

for some $c, C>0$ as claimed. 


\section{Smooth vs. rough projector: proof of Proposition 11}

Let $x_{0} \in M$ be a non-self-focal point and fix $\varepsilon>0$. The proof of Proposition 11 amounts to showing that there exists $c>0$ such that, for all $\varepsilon>0$, there is an open neighborhood $U_{\varepsilon}$ of $x_{0}$ and a positive constant $c_{\varepsilon}$ with

$$
\sup _{x, y \in \mathscr{U}_{\varepsilon}}\left|E_{\lambda}(x, y)-\rho * E_{\lambda}(x, y)\right| \leq c \varepsilon \lambda^{n-1}+c_{\varepsilon} \lambda^{n-2}
$$

for all $\lambda \geq 1$. It is at this point that the assumption that $x_{0}$ is a non-self-focal point is needed. In Section 4 we construct a partition of the identity operator localized to $x_{0}$. We use this partition to split $\left|E_{\lambda}(x, y)-\rho * E_{\lambda}(x, y)\right|$ into different pieces, each of which we shall control using two types of Tauberian theorems, described in Section 6.1. We conclude this section by presenting the proof of Proposition 11 in Section 6.2.

To ease the notation, we will write

$$
E(x, y, \lambda):=E_{\lambda}(x, y) .
$$

To prove (65), we use the operators $B_{\varepsilon}$ and $C_{\varepsilon}$ and the function $\psi_{\varepsilon}$ constructed in Lemma 15 . We set

$$
\begin{aligned}
& \alpha_{\varepsilon}(x, y, \lambda):=E C_{\varepsilon}^{*}(x, y, \lambda)+\frac{1}{2}\left(E(x, x, \lambda)+C_{\varepsilon} E C_{\varepsilon}^{*}(y, y, \lambda)\right), \\
& \beta_{\varepsilon}(x, y, \lambda):=\rho * E C_{\varepsilon}^{*}(x, y, \lambda)+\frac{1}{2}\left(E(x, x, \lambda)+C_{\varepsilon} E C_{\varepsilon}^{*}(y, y, \lambda)\right),
\end{aligned}
$$

where $x$ and $y$ are any two points in $M$. Note that

$$
\left|\alpha_{\varepsilon}(x, y, \lambda)-\beta_{\varepsilon}(x, y, \lambda)\right|=\left|E C_{\varepsilon}^{*}(x, y, \lambda)-\rho * E C_{\varepsilon}^{*}(x, y, \lambda)\right| .
$$

In addition, observe that

$$
\alpha_{\varepsilon}(x, y, \lambda):=\frac{1}{2} \sum_{\lambda_{j} \leq \lambda}\left[\varphi_{j}(x)+\left(C_{\varepsilon} \varphi_{j}\right)(y)\right]^{2}
$$

and so $\alpha_{\varepsilon}(x, y, \lambda)$ is an increasing function of $\lambda$ for any fixed $x$ and $y$. We also set

$$
g_{\varepsilon}(x, y, \lambda):=E B_{\varepsilon}^{*}(x, y, \lambda)-\rho * E B_{\varepsilon}^{*}(x, y, \lambda) .
$$

Since $B_{\varepsilon}+C_{\varepsilon}=\psi_{\varepsilon}^{2}$ and $\psi_{\varepsilon}=1$ in a neighborhood of $x_{0}$, relation (65) would hold if we proved that there exist positive constants $c$ and $c_{\varepsilon}$ with $c$ independent of $\varepsilon$, and a neighborhood $U_{\varepsilon}$ of $x_{0}$, such that, for all $\lambda \geq 1$,

$$
\begin{array}{r}
\sup _{x, y \in \mathscr{U}_{\varepsilon}}\left|\alpha_{\varepsilon}(x, y, \lambda)-\beta_{\varepsilon}(x, y, \lambda)\right| \leq c \varepsilon \lambda^{n-1}+c_{\varepsilon} \lambda^{n-2}, \\
\sup _{x, y \in U_{\varepsilon}}\left|g_{\varepsilon}(x, y, \lambda)\right| \leq c \varepsilon \lambda^{n-1}+c_{\varepsilon} \lambda^{n-2} .
\end{array}
$$

6.1. Tauberian theorems. To control $\left|\alpha_{\varepsilon}(x, y, \lambda)-\beta_{\varepsilon}(x, y, \lambda)\right|$ and $\left|g_{\varepsilon}(x, y, \lambda)\right|$ we use two different Tauberian-type theorems. To state the first one, fix a positive function $\phi \in \mathscr{Y}(\mathbb{R})$ such that supp $\hat{\phi} \subseteq(-1,1)$ and $\hat{\phi}(0)=1$. We have written $\hat{f}$ for the Fourier transform of $f$. Define, for each $a>0$,

$$
\phi_{a}(\lambda):=\frac{1}{a} \phi\left(\frac{\lambda}{a}\right)
$$


so that $\hat{\phi}_{a}(t)=\hat{\phi}(a t)$.

Lemma 19 (Tauberian theorem for monotone functions). Let $\alpha$ be an increasing temperate function with $\alpha(0)=0$ and let $\beta$ be a function of locally bounded variation with $\beta(0)=0$. Suppose further that there exist $M_{0}>0, a>0$ and a constant $c_{a}$ such that:

(a) There exists $m \in \mathbb{N}$ such that

$$
\int_{\mu-a}^{\mu+a}|d \beta| \leq a M_{0}(1+|\mu|)^{m-1}+c_{a}|\mu|^{m-2} \text { for all } \mu \geq 0 .
$$

(b) There exist $\kappa \in \mathbb{Z} \backslash\{-1\}$ with $\kappa \leq m-1$, and $M_{a}>0$, such that

$$
\left|(d \alpha-d \beta) * \phi_{a}(\mu)\right| \leq M_{a}(1+|\mu|)^{\kappa} \quad \text { for all } \mu \geq 0 .
$$

Then there exists $c>0$ depending only on $\phi$ such that

$$
|\alpha(\mu)-\beta(\mu)| \leq c\left(a M_{0}|\mu|^{m-1}+c_{a}|\mu|^{m-2}+M_{a}(1+|\mu|)^{\kappa+1}\right)
$$

for all $\mu \geq 0$.

Proof. The proof is identical to argument for Lemma 17.5.6 in [Hörmander 1985a].

We will also need the following result:

Lemma 20 (Tauberian theorem for nonmonotone functions [Hörmander 1968]). Let $g$ be a piecewise continuous function such that there exists $a>0$ with $\hat{g}(t) \equiv 0$ for $|t| \leq a$. Suppose further that, for all $\mu \in \mathbb{R}$, there exist constants $m \in \mathbb{N}$ and $c_{1}, c_{2}>0$ such that

$$
|g(\mu+s)-g(\mu)| \leq c_{1}(1+|\mu|)^{m}+c_{2}(1+|\mu|)^{m-1} \quad \text { for all } s \in[0,1] .
$$

Then there exists a positive constant $c_{m, a}$, depending only on $m$ and $a$, such that, for all $\mu$,

$$
|g(\mu)| \leq c_{m, a}\left(c_{1}(1+|\mu|)^{m}+c_{2}(1+|\mu|)^{m-1}\right) .
$$

6.2. Proof of Proposition 11. As explained above, the proof of Proposition 11 reduces to establishing relations (69) and (70).

Proof of (69). We seek to apply Lemma 19 to $\alpha_{\varepsilon}$ and $\beta_{\varepsilon}$. Let $a=\varepsilon, m=n$ and $\kappa=-2$. We first verify condition (a). From Remark 17, it follows that there exist an open neighborhood $u_{\varepsilon}$ of $x_{0}$ and constants $c_{1}, c_{\varepsilon}>0$ such that, for all $x, y \in U_{\varepsilon}$ and all $\lambda \geq 1$,

$$
\begin{aligned}
\int_{\lambda-\varepsilon}^{\lambda+\varepsilon}\left(\left|\partial_{\nu} E(x, x, v)\right|+\left|\partial_{\nu}\left(C_{\varepsilon} E C_{\varepsilon}^{*}\right)(y, y, v)\right|\right) d v & =\sum_{\left|\lambda_{j}-\lambda\right| \leq \varepsilon}\left(\varphi_{j}(x)\right)^{2}+\left(C_{\varepsilon} \varphi_{j}(y)\right)^{2} \\
& \leq c_{1} \varepsilon \lambda^{n-1}+c_{\varepsilon} \lambda^{n-2}
\end{aligned}
$$

Combining (74) with the estimate in Proposition 18 applied to $Q=C_{\varepsilon}$, we see that there exist positive constants $M_{0}$ and $c_{\varepsilon}$ for which

$$
\sup _{x, y \in \cup_{\varepsilon}} \int_{\lambda-\varepsilon}^{\lambda+\varepsilon}\left|\partial_{\nu} \beta_{\varepsilon}(x, y, v)\right| d \nu \leq M_{0} \varepsilon \lambda^{n-1}+c_{\varepsilon} \lambda^{n-2}
$$


for all $\lambda \geq 1$. It remains to verify condition (b). Note that

$$
\partial_{\lambda}\left(\alpha_{\varepsilon}(x, y, \cdot)-\beta_{\varepsilon}(x, y, \cdot)\right) * \phi_{\varepsilon}(\lambda)=\mathscr{F}_{t \rightarrow \lambda}^{-1}\left((1-\hat{\rho}(t)) \hat{\phi}_{\varepsilon}(t)\left(U(t) C_{\varepsilon}^{*}\right)(x, y)\right)(\lambda),
$$

where $\mathscr{F}$ is the Fourier transform and $\phi_{\varepsilon}$ is defined in (71). According to Lemma $15, U(t) C_{\varepsilon}^{*}$ is a smoothing operator for $\frac{1}{2} \operatorname{inj}(M, g)<|t|<\frac{1}{\varepsilon}$. Hence, since

$$
\operatorname{supp} \hat{\phi}_{\varepsilon} \subset\left\{t:|t|<\frac{1}{\varepsilon}\right\} \quad \text { and } \quad \operatorname{supp}(1-\hat{\rho}) \subset\left\{t:|t|>\frac{1}{2} \operatorname{inj}(M, g)\right\},
$$

we find that, for each $N$, there are constants $c_{N, \varepsilon}$ depending on $N$ and $\varepsilon$ that satisfy

$$
\sup _{x, y \in M}\left|\partial_{\lambda}\left(\alpha_{\varepsilon}(x, y, \cdot)-\beta_{\varepsilon}(x, y, \cdot)\right) * \phi_{\varepsilon}(\lambda)\right| \leq c_{N, \varepsilon}(1+|\lambda|)^{-N}
$$

for all $\lambda>0$.

Proof of (70). We seek to apply Lemma 20 to $g_{\varepsilon}$. First, note that, since

$$
g_{\varepsilon}(x, y, \lambda)=E B_{\varepsilon}^{*}(x, y, \lambda)-\rho * E B_{\varepsilon}^{*}(x, y, \lambda),
$$

the function $g_{\varepsilon}(x, y, \cdot)$ is piecewise continuous in the $\lambda$ variable. Next, we check that $\hat{g_{\varepsilon}}(t) \equiv 0$ in a neighborhood of $t=0$. We have

$$
\partial_{\lambda} g_{\varepsilon}(x, y, \lambda)=\mathscr{F}_{t \rightarrow \lambda}^{-1}\left((1-\hat{\rho}(t))\left(U(t) B_{\varepsilon}^{*}\right)(x, y)\right)(\lambda) .
$$

Since $\hat{\rho} \equiv 1$ on $\left(-\frac{1}{2} \operatorname{inj}(M, g), \frac{1}{2} \operatorname{inj}(M, g)\right)$, we have $\mathscr{F}_{\lambda \rightarrow t}\left(\partial_{\lambda} g_{\varepsilon}(x, y, \cdot)\right)(t)=0$ for $|t| \leq \frac{1}{2} \operatorname{inj}(M, g)$. Equivalently,

$$
t \cdot \mathscr{F}_{\lambda \rightarrow t}\left(g_{\varepsilon}(x, y, \cdot)\right)(t)=0, \quad|t| \leq \frac{1}{2} \operatorname{inj}(M, g) .
$$

In addition, we must have $\mathscr{F}_{\lambda \rightarrow t}\left(g_{\varepsilon}(x, y, \cdot)\right)(0)=0$, for otherwise $g_{\varepsilon}(x, y, \cdot)$ would include a sum of derivatives of delta functions but this is not possible, since $g_{\varepsilon}(x, y, \cdot)$ is piecewise continuous. It follows that

$$
\mathscr{F}_{\lambda \rightarrow t}\left(g_{\varepsilon}(x, y, \cdot)\right)(t)=0, \quad|t| \leq \frac{1}{2} \operatorname{inj}(M, g),
$$

as desired. It therefore remains to check that $g_{\varepsilon}$ satisfies (73). Let $s \in[0,1], \lambda \in \mathbb{R}$ and write

$$
\begin{aligned}
g_{\varepsilon}(x, y, \lambda+s) & -g_{\varepsilon}(x, y, \lambda) \\
& =E B_{\varepsilon}^{*}(x, y, \lambda+s)-E B_{\varepsilon}^{*}(x, y, \lambda)+\rho * E B_{\varepsilon}^{*}(x, y, \lambda+s)-\rho * E B_{\varepsilon}^{*}(x, y, \lambda) .
\end{aligned}
$$

To estimate $E B_{\varepsilon}^{*}(x, y, \lambda+s)-E B_{\varepsilon}^{*}(x, y, \lambda)$ we apply the Cauchy-Schwarz inequality:

$$
\begin{aligned}
E B_{\varepsilon}^{*}(x, y, \lambda+s)-E B_{\varepsilon}^{*}(x, y, \lambda) & =\sum_{\lambda \leq \lambda_{j} \leq \lambda+s} \varphi_{j}(x) B_{\varepsilon} \varphi_{j}(y) \\
& \leq\left(\sum_{\lambda \leq \lambda_{j} \leq \lambda+s}\left(\varphi_{j}(x)\right)^{2}\right)^{\frac{1}{2}}\left(\sum_{\lambda \leq \lambda_{j} \leq \lambda+s}\left(B_{\varepsilon} \varphi_{j}(y)\right)^{2}\right)^{\frac{1}{2}} .
\end{aligned}
$$


Applying Remark 17 to $Q=\mathrm{Id}$ and $Q=B_{\varepsilon}$, there exist an open neighborhood $U_{\varepsilon}$ of $x_{0}$ and constants $c, c_{\varepsilon}>0$ such that

$$
\left|E B_{\varepsilon}^{*}(x, y, \lambda+s)-E B_{\varepsilon}^{*}(x, y, \lambda)\right| \leq c \varepsilon \lambda^{n-1}+c_{\varepsilon} \lambda^{n-2}
$$

for all $\lambda \geq 1, s \in[0,1]$ and $x, y \in \cup_{\varepsilon}$. The $\varepsilon$ factor is due to the fact that $\left\|b_{0}\right\|_{1}<\varepsilon$.

To estimate $\rho * E B_{\varepsilon}^{*}(x, y, \lambda+s)-\rho * E B_{\varepsilon}^{*}(x, y, \lambda)$ we apply Proposition 18 to the operator $Q=B_{\varepsilon}$. Since there exists $\tilde{c}>0$ with

$$
\left|\partial_{\lambda} \rho * E B_{\varepsilon}^{*}(x, y, \lambda)\right| \leq \tilde{c}\left(\left\|b_{0}\right\|_{1} \lambda^{n-1}+\lambda^{n-2}\right) \quad \text { for all } \lambda \geq 1
$$

and $\left\|b_{0}\right\|_{1} \leq \varepsilon$, we get (after possibly enlarging $c$ and $c_{\varepsilon}$ ) that

$$
\left|\rho * E B_{\varepsilon}^{*}(x, y, \lambda+s)-\rho * E B_{\varepsilon}^{*}(x, y, \lambda)\right| \leq c \varepsilon \lambda^{n-1}+c_{\varepsilon} \lambda^{n-2} \text { for all } \lambda \geq 1 .
$$

Combining (76) and (77) into (75), we conclude the existence of positive constants $c$ and $c_{\varepsilon}$ such that

$$
\left|g_{\varepsilon}(x, y, \lambda+s)-g_{\varepsilon}(x, y, \lambda)\right| \leq c \varepsilon \lambda^{n-1}+c_{\varepsilon} \lambda^{n-2} \text { for all } \lambda \geq 1
$$

and $s \in[0,1]$, as desired. Applying Lemma 20 with $m=n$ and $a=\frac{1}{2} \operatorname{inj}(M, g)$ proves (70).

\section{Proof of Theorems 6-9}

Proof of Theorem 6. Suppose that $(M, g)$ is a smooth, compact, Riemannian manifold with no boundary. Let $K \subseteq M \times M$ be a compact set satisfying that any pair of points in it are mutually nonfocal. We aim to show that there exists $c>0$ such that, for every $\varepsilon>0$, there are constants $\lambda_{\varepsilon}>0$ and $c_{\varepsilon}>0$ such that

$$
\sup _{(x, y) \in K}|R(x, y, \lambda)| \leq c \varepsilon \lambda^{n-1}+c_{\varepsilon} \lambda^{n-2}
$$

for all $\lambda>\lambda_{\varepsilon}$. Fix $\varepsilon>0$ and write $\Delta \subseteq M \times M$ for the diagonal. Define

$$
\widetilde{K}=K \cap \Delta \text {. }
$$

By (16), there exists $\lambda_{\varepsilon}>0$, a finite collection $\left\{x_{j}: j=1, \ldots, N_{\varepsilon}\right\}$ and open neighborhoods $\mathscr{U}_{\varepsilon}^{x_{j}}$ of $x_{j}$ such that

$$
\widetilde{K} \subseteq \bigcup_{j} u_{\varepsilon}^{x_{j}} \times u_{\varepsilon}^{x_{j}}
$$

and

for all $\lambda>\lambda_{\varepsilon}$. Define

$$
\sup _{x, y \in \cup_{\varepsilon}^{x_{j}}}|R(x, y, \lambda)| \leq c \varepsilon \lambda^{n-1}+c_{\varepsilon} \lambda^{n-2}
$$

$$
K_{\varepsilon}:=K \backslash \bigcup_{j} u_{\varepsilon}^{x_{j}} \times u_{\varepsilon}^{x_{j}} .
$$

Safarov [1988, Theorem 3.3] proved under the mutually nonfocal assumption that

$$
\sup _{(x, y) \in K_{\varepsilon}}|R(x, y, \lambda)|=o_{\varepsilon}\left(\lambda^{n-1}\right) .
$$


Combining (78) and (79) completes the proof.

Proof of Theorem 8 . The injectivity of the maps $\Psi_{(\lambda, \lambda+1]}: M \rightarrow \mathbb{R}^{m_{\lambda}}$ for $\lambda$ large enough is implied by the existence of positive constants $c_{1}, c_{2}, r_{0}$ and $\lambda_{r_{0}}$ such that, if $\lambda>\lambda_{r_{0}}$, then

$$
\inf _{x, y: \lambda \operatorname{dist} g(x, y) \geq r_{0}} \operatorname{dist}_{\lambda}^{2}(x, y)>c_{1}
$$

and

$$
\inf _{x, y: \lambda \operatorname{distg}_{g}(x, y)<r_{0}} \frac{\operatorname{dist}_{\lambda}^{2}(x, y)}{\lambda^{2} \operatorname{dist}_{g}(x, y)^{2}}>c_{2} .
$$

We first prove (80). By Theorem 6, for all $x, y \in M$,

$$
\operatorname{dist}_{\lambda}^{2}(x, y)=f\left(\lambda \operatorname{dist}_{g}(x, y)\right)+\widetilde{R}(x, y, \lambda),
$$

where $\sup _{x, y \in M}|\widetilde{R}(x, y, \lambda)|=o(1)$ and $f:[0,+\infty) \rightarrow \mathbb{R}$ is the function

$$
f(r):=\int_{S^{n-1}}\left(1-e^{i r \omega_{1}}\right) d \omega .
$$

Observe that $f(r) \geq 0$, with $f(r)=0$ only if $r=0$. Moreover,

$$
f(r)=\sigma_{n}+O\left(r^{-(n-1) / 2}\right) \quad \text { as } r \rightarrow \infty \quad \text { and } \quad f(r)=r^{2} \cdot \tilde{f}(r)
$$

for some smooth and positive function $\tilde{f}$, where $\sigma_{n}$ is the volume of $S^{n-1}$. According to the first relation in (83), we may choose $r_{0}>0$ so that

$$
\lambda \operatorname{dist}_{g}(x, y) \geq r_{0} \Longrightarrow\left|f\left(\lambda \operatorname{dist}_{g}(x, y)\right)-\sigma_{n}\right| \leq \frac{1}{4} \sigma_{n} .
$$

Moreover, by Theorem 6 we may choose $\lambda_{r_{0}}$ so that, if $\lambda>\lambda_{r_{0}}$, then

$$
\sup _{x, y \in M}|\tilde{R}(x, y, \lambda)| \leq \frac{1}{4} \sigma_{n} .
$$

Combining (82), (84) and (85), we find that, for all $\lambda>\lambda_{r_{0}}$ and all $x, y \in M$ with $\lambda \operatorname{dist}_{g}(x, y) \geq r_{0}$,

$$
\operatorname{dist}_{\lambda}^{2}(x, y) \geq \frac{1}{2} \sigma_{n},
$$

as desired. To verify (81), write, as above,

$$
\operatorname{dist}_{\lambda}^{2}(x, y)=\frac{(2 \pi)^{n}}{2 \lambda^{n-1}}\left(E_{(\lambda, \lambda+1]}(x, x)+E_{(\lambda, \lambda+1]}(y, y)-2 E_{(\lambda, \lambda+1]}(x, y)\right)
$$

and note that the first derivatives of $\operatorname{dist}_{\lambda}^{2}(x, y)$ in $x$ and $y$ all vanish when $x=y$. Moreover, by [Zelditch 2009, Proposition 2.3], we have that the Hessian of $E_{(\lambda, \lambda+1]}$ may be written as

$$
\left.d_{x} \otimes d_{y}\right|_{x=y} E_{(\lambda, \lambda+1]}(x, y)=C_{n} \lambda^{n+1} g_{x}+o\left(\lambda^{n+1}\right),
$$

where $g_{x}$ is the metric $g$ on $T_{x} M$, and Equation (1.2) in [Potash 2014] shows that

$$
C_{n}=\frac{\sigma_{n}}{n(2 \pi)^{n}}
$$


Therefore, applying Taylor's theorem, there exists $C_{0}>0$ for which

$$
\left|\frac{\operatorname{dist}_{\lambda}^{2}(x, y)}{\lambda^{2} \operatorname{dist}_{g}^{2}(x, y)}-\frac{\sigma_{n}}{2 n}\right| \leq C_{0} \cdot \lambda \operatorname{dist}_{g}(x, y) .
$$

The extra factor of $\lambda$ on the right-hand side of (86) comes from the fact that

$$
\sup _{|\alpha|=3}\left|\partial_{x}^{\alpha}\right|_{x=y} E_{(\lambda, \lambda+1]}(x, y) \mid=O\left(m_{\lambda} \lambda^{3}\right)
$$

which is proved, for example, in [Xu 2006, Equation (2.7)]. Equation (86) shows that

$$
\inf _{\lambda \operatorname{dist}_{g}(x, y)<\sigma_{n} /\left(4 n C_{0}\right)} \frac{\operatorname{dist}_{\lambda}^{2}(x, y)}{\lambda^{2} d_{g}^{2}(x, y)} \geq \frac{\sigma_{n}}{2 n}>0 .
$$

If $r_{0} \leq \sigma_{n} /\left(4 n C_{0}\right)$, then the claim (81) follows. Otherwise, it remains to show that there exists $c_{2}>0$ with

$$
\inf _{\sigma_{n} /\left(4 n C_{0}\right) \leq \lambda \operatorname{dist}_{g}(x, y)<r_{0}} \frac{\operatorname{dist}_{\lambda}^{2}(x, y)}{\lambda^{2} d_{g}^{2}(x, y)}>c_{2}
$$

for all $\lambda$ sufficiently large. Theorem 6 shows that, after possibly enlarging $\lambda_{r_{0}}$, we have

$$
\sup _{x, y \in M}|\widetilde{R}(x, y, \lambda)| \leq\left(\frac{\sigma_{n}}{4 n C_{0}}\right)^{2} \inf _{r<r_{0}} \tilde{f}(r)
$$

for all $\lambda>\lambda r_{0}$. Then the second relation in (83) combined with (82) yields that, for all $\lambda>\lambda r_{0}$,

$$
\inf _{\sigma_{n} /\left(4 n C_{0}\right) \leq \lambda \operatorname{dist}_{g}(x, y)<r_{0}} \operatorname{dist}_{\lambda}^{2}(x, y) \geq\left(\frac{\sigma_{n}}{4 n C_{0}}\right)^{2} \inf _{r<r_{0}} \tilde{f}(r)>0 .
$$

This completes the proof of (81).

Proof of Theorem 9. By (13) and Theorem 6 we have that

$$
\sup _{x, y \in M}\left|\operatorname{dist}_{\lambda}^{2}(x, y)-\int_{S^{n-1}}\left(1-e^{i \lambda \operatorname{dist}_{g}(x, y) \omega_{1}}\right) d \omega\right|=o(1)
$$

as $\lambda \rightarrow \infty$. Combining this with

$$
\frac{1}{\lambda^{2} \operatorname{dist}_{g}(x, y)^{2}} \int_{S^{n-1}}\left(1-e^{i \lambda \operatorname{dist}_{g}(x, y) \omega_{1}}\right) d \omega=\frac{\sigma_{n}}{2 n}+O\left(\lambda^{2} \operatorname{dist}_{g}^{2}(x, y)\right)
$$

and (86) completes the proof.

\section{Acknowledgements}

It is our pleasure to thank I. Polterovich, C. Sogge, J. Toth and particularly Y. Safarov and S. Zelditch for providing detailed comments on earlier drafts of this article. We are also grateful to an anonymous referee whose suggestions and corrections significantly improved the exposition. In particular, the referee pointed us to [Laptev et al. 1994], simplifying our previous parametrix construction in Section 3, and 
exposed an error in the original version of Proposition 18. Fixing our mistake ultimately lead to a more efficient proof. Canzani would also like to thank B. Xu for sharing unpublished proofs of some results in [Xu 2006].

\section{References}

[Bérard 1977] P. H. Bérard, "On the wave equation on a compact Riemannian manifold without conjugate points", Math. Z. 155:3 (1977), 249-276. MR 56 \#13295 Zbl 0341.35052

[Bérard et al. 1994] P. Bérard, G. Besson, and S. Gallot, "Embedding Riemannian manifolds by their heat kernel", Geom. Funct. Anal. 4:4 (1994), 373-398. MR 95g:58228 Zbl 0806.53044

[Berger et al. 1971] M. Berger, P. Gauduchon, and E. Mazet, Le spectre d'une variété riemannienne, Lecture Notes in Mathematics 194, Springer, Berlin, 1971. MR 43 \#8025 Zbl 0223.53034

[Colin de Verdière 1980] Y. Colin de Verdière, "Spectre conjoint d'opérateurs pseudo-différentiels qui commutent, II: Le cas intégrable", Math. Z. 171:1 (1980), 51-73. MR 81i:58046

[Duistermaat and Guillemin 1975] J. J. Duistermaat and V. W. Guillemin, "The spectrum of positive elliptic operators and periodic bicharacteristics", Invent. Math. 29:1 (1975), 39-79. MR 53 \#9307 Zbl 0307.35071

[Helgason 1981] S. Helgason, Topics in harmonic analysis on homogeneous spaces, Progress in Mathematics 13, Birkhäuser, Boston, 1981. MR 83g:43009 Zbl 0467.43001

[Hörmander 1968] L. Hörmander, "The spectral function of an elliptic operator”, Acta Math. 121 (1968), 193-218. MR 58 \#29418 Zbl 0164.13201

[Hörmander 1985a] L. Hörmander, The analysis of linear partial differential operators, III: Pseudodifferential operators, Grundlehren der Math. Wissenschaften 274, Springer, Berlin, 1985. MR 87d:35002a Zbl 0601.35001

[Hörmander 1985b] L. Hörmander, The analysis of linear partial differential operators, IV: Fourier integral operators, Grundlehren der Math. Wissenschaften 275, Springer, Berlin, 1985. MR 87d:35002b Zbl 0612.35001

[Ivriĭ 1984] V. Ivriǔ, Precise spectral asymptotics for elliptic operators acting in fiberings over manifolds with boundary, Lecture Notes in Mathematics 1100, Springer, Berlin, 1984. MR 86h:58139 Zbl 0565.35002

[Jakobson and Polterovich 2007] D. Jakobson and I. Polterovich, "Estimates from below for the spectral function and for the remainder in local Weyl's law", Geom. Funct. Anal. 17:3 (2007), 806-838. MR 2009h:35302 Zbl 1161.58012

[Jones et al. 2008] P. W. Jones, M. Maggioni, and R. Schul, "Manifold parametrizations by eigenfunctions of the Laplacian and heat kernels", Proc. Natl. Acad. Sci. USA 105:6 (2008), 1803-1808. MR 2008m:58053 Zbl 1215.58012

[Lapointe et al. 2009] H. Lapointe, I. Polterovich, and Y. Safarov, "Average growth of the spectral function on a Riemannian manifold", Comm. Partial Differential Equations 34:4-6 (2009), 581-615. MR 2011e:58050 Zbl 1177.58020

[Laptev et al. 1994] A. Laptev, Y. Safarov, and D. Vassiliev, "On global representation of Lagrangian distributions and solutions of hyperbolic equations", Comm. Pure Appl. Math. 47:11 (1994), 1411-1456. MR 95m:58121 Zbl 0811.35177

[Nicolaescu 2012] L. Nicolaescu, "The blowup along the diagonal of the spectral function of the Laplacian", preprint, 2012. arXiv 1103.1276

[Petridis and Toth 2002] Y. N. Petridis and J. A. Toth, "The remainder in Weyl's law for Heisenberg manifolds", J. Differential Geom. 60:3 (2002), 455-483. MR 2004c:58054 Zbl 1066.58017

[Potash 2014] E. Potash, "Euclidean embeddings and Riemannian Bergman metrics", preprint, 2014. To appear in J. Geom. Anal. arXiv 1310.4878

[Randol 1981] B. Randol, "A Dirichlet series of eigenvalue type with applications to asymptotic estimates", Bull. London Math. Soc. 13:4 (1981), 309-315. MR 82j:10082 Zbl 0458.30029

[Safarov 1988] Y. G. Safarov, "Asymptotics of a spectral function of a positive elliptic operator without a nontrapping condition", Funktsional. Anal. i Prilozhen. 22:3 (1988), 53-65, 96. In Russian; translated in Funct. Anal. Appl. 22:3 (1988), $213-223$. MR 89i:35108 Zbl 0679.35074

[Safarov and Vassiliev 1997] Y. Safarov and D. Vassiliev, The asymptotic distribution of eigenvalues of partial differential operators, Translations of Mathematical Monographs 155, Amer. Math. Soc., Providence, RI, 1997. MR 97h:58163 Zbl 0898.35003 
[Sarnak and Wigman 2014] P. Sarnak and I. Wigman, "Topologies of nodal sets of random band limited functions", preprint, 2014. arXiv 1312.7858

[Sodin 2012] M. Sodin, "Lectures on random nodal portraits", lecture notes, St. Petersburg Summer School in Probability and Statistical Physics, 2012, available at http://www.math.tau.ac.il/ sodin/SPB-Lecture-Notes.pdf.

[Sogge 1993] C. D. Sogge, Fourier integrals in classical analysis, Cambridge Tracts in Mathematics 105, Cambridge University Press, Cambridge, 1993. MR 94c:35178 Zbl 0783.35001

[Sogge and Zelditch 2002] C. D. Sogge and S. Zelditch, "Riemannian manifolds with maximal eigenfunction growth", Duke Math. J. 114:3 (2002), 387-437. MR 2004b:58053 Zbl 1018.58010

[Sogge and Zelditch 2013] C. Sogge and S. Zelditch, "Focal points and sup-norms of eigenfunctions", preprint, 2013. arXiv 1311.3999

[Szegő 1975] G. Szegó, Orthogonal polynomials, 4th ed., AMS Colloquium Publications 23, Amer. Math. Soc., Providence, RI, 1975. MR 51 \#8724 Zbl 0305.42011

[Xu 2006] B. Xu, "Asymptotic behavior of $L^{2}$-normalized eigenfunctions of the Laplace-Beltrami operator on a closed Riemannian manifold", pp. 99-117 in Harmonic analysis and its applications, edited by A. Miyachi et al., Yokohama Publ., Yokohama, 2006. MR 2007b:58044 Zbl 1238.58021

[Zelditch 1997] S. Zelditch, "Fine structure of Zoll spectra", J. Funct. Anal. 143:2 (1997), 415-460. MR 98g:58173 Zbl 0870.58103

[Zelditch 2001] S. Zelditch, "From random polynomials to symplectic geometry", pp. 367-376 in XIII-th International Congress on Mathematical Physics (London, 2000), edited by A. Grigoryan et al., Int. Press, Boston, 2001. MR 2003a:32034 Zbl 1076.32016

[Zelditch 2008] S. Zelditch, "Local and global analysis of eigenfunctions on Riemannian manifolds", pp. 545-658 in Handbook of geometric analysis, I, edited by L. Ji et al., Adv. Lect. Math. 7, International Press, 2008. MR 2010b:58040 Zbl 1176.58017

[Zelditch 2009] S. Zelditch, "Real and complex zeros of Riemannian random waves", pp. 321-342 in Spectral analysis in geometry and number theory, edited by M. Kotani et al., Contemp. Math. 484, Amer. Math. Soc., Providence, RI, 2009. MR 2010c:58040 Zbl 1176.58021

Received 3 Feb 2015. Revised 2 Jun 2015. Accepted 31 Jul 2015.

YAIZA CANZANI: canzani@math.harvard.edu

Harvard Mathematics Department, Harvard University, Cambridge, MA 02138, United States

BORIS HANIN: bhanin@mit.edu

Department of Mathematics, Massachussetts Institute of Technology, Cambridge, MA 02139, United States 


\title{
Analysis \& PDE
}

\author{
msp.org/apde
}

\section{EDITORS}

EDITOR-IN-CHIEF

\author{
Maciej Zworski \\ zworski@math.berkeley.edu \\ University of California \\ Berkeley, USA
}

BOARD OF EDITORS

Nicolas Burq Université Paris-Sud 11, France

nicolas.burq@math.u-psud.fr

Sun-Yung Alice Chang Princeton University, USA

chang@math.princeton.edu

Michael Christ University of California, Berkeley, USA

mchrist@math.berkeley.edu

Charles Fefferman Princeton University, USA

cf@math.princeton.edu

Ursula Hamenstaedt Universität Bonn, Germany

ursula@math.uni-bonn.de

Vaughan Jones U.C. Berkeley \& Vanderbilt University vaughan.f.jones@vanderbilt.edu

Herbert Koch Universität Bonn, Germany koch@math.uni-bonn.de

Izabella Laba University of British Columbia, Canada ilaba@math.ubc.ca

Gilles Lebeau Université de Nice Sophia Antipolis, France lebeau@unice.fr

László Lempert Purdue University, USA lempert@math.purdue.edu

Richard B. Melrose Massachussets Institute of Technology, USA rbm@math.mit.edu

Frank Merle Université de Cergy-Pontoise, France Frank.Merle@u-cergy.fr

William Minicozzi II Johns Hopkins University, USA minicozz@math.jhu.edu

Werner Müller Universität Bonn, Germany mueller@math.uni-bonn.de
Yuval Peres

Gilles Pisier

Tristan Rivière

Igor Rodnianski

Wilhelm Schlag

Sylvia Serfaty

Yum-Tong Siu

Terence Tao

Michael E. Taylor

Gunther Uhlmann

András Vasy

Dan Virgil Voiculescu

Steven Zelditch
University of California, Berkeley, USA

peres@stat.berkeley.edu

Texas A\&M University, and Paris 6

pisier@math.tamu.edu

ETH, Switzerland

riviere@math.ethz.ch

Princeton University, USA

irod@math.princeton.edu

University of Chicago, USA

schlag@math.uchicago.edu

New York University, USA

serfaty@cims.nyu.edu

Harvard University, USA siu@math.harvard.edu

University of California, Los Angeles, USA tao@math.ucla.edu

Univ. of North Carolina, Chapel Hill, USA met@math.unc.edu

University of Washington, USA gunther@math.washington.edu

Stanford University, USA andras@math.stanford.edu

University of California, Berkeley, USA

dvv@math.berkeley.edu

Northwestern University, USA

zelditch@math.northwestern.edu

PRODUCTION

production@msp.org

Silvio Levy, Scientific Editor

See inside back cover or msp.org/apde for submission instructions.

The subscription price for 2015 is US \$205/year for the electronic version, and \$390/year ( $\$ 55$, if shipping outside the US) for print and electronic. Subscriptions, requests for back issues from the last three years and changes of subscribers address should be sent to MSP.

Analysis \& PDE (ISSN 1948-206X electronic, 2157-5045 printed) at Mathematical Sciences Publishers, 798 Evans Hall \#3840, c/o University of California, Berkeley, CA 94720-3840, is published continuously online. Periodical rate postage paid at Berkeley, CA 94704, and additional mailing offices.

APDE peer review and production are managed by EditFLOw ${ }^{\circledR}$ from MSP.

\section{PUBLISHED BY}

mathematical sciences publishers

nonprofit scientific publishing

http://msp.org/

(C) 2015 Mathematical Sciences Publishers 


\section{ANALYSIS \& PDE}

\section{Volume $8 \quad$ No. $7 \quad 2015$}

A pointwise inequality for the fourth-order Lane-Emden equation

Mostafa FaZly, Jun-Cheng Wei and Xingwang XU

Convergence rates and Hölder estimates in almost-periodic homogenization of elliptic systems 1565 ZHONGWEI SHEN

Quantitative decay rates for dispersive solutions to the Einstein-scalar field system in spherical 1603 symmetry

JONATHAN LUK and SUNG-JIN OH

A model for studying double exponential growth in the two-dimensional Euler equations

NETS KATZ and ANDREW TAPAY

Asymptotics of Hadamard type for eigenvalues of the Neumann problem on $C^{1}$-domains for 1695 elliptic operators

JOHAN THIM

Scaling limit for the kernel of the spectral projector and remainder estimates in the pointwise 1707 Weyl law

YAIZA CANZANI and BORIS HANIN

On the continuous resonant equation for NLS, II: Statistical study

PIERRE GERMAIN, ZAHER HANI and LAURENT THOMANN

Blow-up analysis of a nonlocal Liouville-type equation

FRANCESCA DA LIO, LUCA MARTINAZZI and TRISTAN RIVIÈRE 